\title{
Neuroindices of cognitive workload: neuroimaging, pupillometric and event-related potential studies of brain work
}

\author{
Marcel Adam Just $\dagger^{*}$, Patricia A. Carpenter $\dagger$ and Akira Miyake $\dagger$ \\ $\uparrow$ Carnegie Mellon University, Pittsburgh, PA 15213, USA \\ $\$$ University of Colorado at Boulder, Boulder, CO, USA
}

Keywords: Brain imaging; cognitive modeling; ERP; fMRI; mental rotation; pupillary dilation.

\begin{abstract}
This article is a selective theory-driven review that synthesizes recent neuroscience findings concerning mental workload during complex cognition from the perspective of a functional resource theory called 3CAPS, focusing on the concept of capacity utilization. Capacity utilization refers to the proportion of resources that is being consumed in a given time interval in a given cognitive system. This definition integrates the dynamic effects of (a) the computational demand imposed by a task, and (b) the resource supply in an individual that is available to meet that demand. The analysis reveals that the functional relations between capacity utilization and measures of neural activity are similar across three different cognitive systems (language comprehension, visuospatial processing and executive processing). The measures of neural activity include functional neuroimaging, pupillary dilation and event-related potentials. The construct of capacity utilization provides a mapping between a functional architecture of cognition and aspects of its neural implementation.
\end{abstract}

\section{Introduction}

That thinking is information processing is the fundamental insight of the cognitive revolution, but it fails to directly address one of the key issues in ergonomics, namely cognitive workload. Information processing theories of cognition often focus on representations and algorithms in order to abstractly characterize the transformation of information from input to output. This simplification is useful but neglects to acknowledge that thinking is biological work and, as in any biological system, the activity entails a consumption of a limited pool of resources. This activity can occur at various levels of intensity, up to some upper limit. From an ergonomic perspective, it is important to empirically and theoretically characterize this intensity dimension of thought, to be able to say what it means to be 'thinking harder'. Purely behavioural measures fail to provide a fully satisfactory index of this aspect of cognition, hence the need for neuroindices of cognition. This paper develops a theoretical framework that relates cognitive workload to resource consumption and availability, and describes results from a variety of studies that show a systematic relation between cognitive workload and several physiological measures of cognitive workload, namely functional neuroimaging measures, pupillary dilation and event-related potentials (ERPs).

A central goal of this article is to propose an explicit mapping between the workings of the cognitive system at the functional level and the amount of activity

$\dagger$ Author for correspondence. e-mail: just@cmu.edu

Theoretical Issues in Ergonomics Science ISSN 1463-922X print/ISSN 1464-536X online (C) 2003 Taylor \& Francis Ltd http://www.tandf.co.uk/journals 
in the underlying cortical neural systems during immediate thought. By immediate thought, we refer to cognitive processes that tend to be relatively brief (less than $30 \mathrm{~s}$ ) and involve novel computations, such as the processes in comprehending sentences, manipulating visual images and solving problems. These processes can be contrasted with others that are currently outside the scope of the theory, including the acquisition or retrieval of declarative knowledge and low-level perceptual and motor processes. The goal of the article is to provide some unifying principles concerning resource consumption at the functional and neural levels across several cognitive domains.

In mapping between cognitive function and neural processing, we examine three different measures of neural activity: functional neuroimaging measures, pupillary dilation and event related potentials (ERPs). Although each measure has a technology and methodology of its own, we suggest that they can be viewed as indices of neural activation and resource consumption; hence, they can be interpreted in terms of a theory in which cognitive activity entails a dynamic modulation of resource consumption. Thus, this theory-driven review paper integrates several findings using disparate types of dependent measures to reveal the high-level similarities across the domains of language comprehension, visuospatial processing and problem solving.

This paper begins by briefly summarizing a cognitive theory of resource consumption and its relation to the underlying neural systems. This theory, specified within a computational architecture called 3CAPS (Just and Carpenter 1992, Just and Varma 2002), proposes that cognitive processes are modulated by the amount and type of resources demanded in a task relative to the supply of those resources that the individual has available. To analyze the effect of resource demand, we will consider studies that manipulate task demands. To analyze the effect of resource supply, we will consider variations in individual differences in processing skill among normal adults. After the description of 3CAPS, we examine how resource demand and supply may relate to indices of neural processing in three cognitive systems: language, spatial and executive processing.

\section{A resource theory of working memory systems}

Previous resource theories, typically developed to account for low-level attention tasks, refer to some underlying commodity that enables performance, usually labelling it either 'capacity' or 'resources' (Kahneman 1973, Norman and Bobrow 1975, Navon and Gopher 1979, Wickens 1984). Their central assumption was that more difficult tasks consume more resources. Although these earlier theories met some success in cognitive psychology and ergonomics, they shared two major problems (Navon 1984): (a) failing to define the resource independently of the behavioural or brain activity outcome, and (b) failing to specify how the resource is consumed online by the cognitive system to support dynamic cognitive activities. The computationally-based resource theory that we briefly describe here, called 3CAPS, addresses and overcomes both of these challenges (Just and Carpenter 1992).

\subsection{Basic mechanisms and assumptions of the theory}

The fundamental assumption of the current theory is that immediate thought is supported by a set of limited, system-specific activation resources that enable the maintenance of representations and the cognitive operations themselves. At a macrolevel, the entire cognitive system is composed of several major interconnected systems, each of which is supported by its own activation resource pool. Within a 
system, such as the language processing system, demand for activation resources arises from keeping the activation levels of needed representational elements above some threshold level and from executing computations, which entails propagating additional activation to selected elements. The computations are performed by activation-based production rules (if-then rules or condition-action contingencies). As long as the condition elements of a rule have an adequately high activation level, then the rule 'fires' by propagating activation to its action elements, possibly for several iterations. A parsing production rule, for example, might have as its condition the encoding of a definite article (the) and, as its action, increasing the activation associated with the representation of a noun phrase.

Basic to 3CAPS is the idea that the supply of the activation resources within a system is limited. If the productions require a large amount of activation (relative to the supply) to complete their functions or if there are too many elements to keep activated in the face of demanding computations, then the demands on the resource pool may begin to outstrip the supply. In such cases, less activation will be propagated per cycle to each action element than would otherwise be the case. The propagation of activation is scaled back in proportion to the amount of activation shortage. This slows the processing rate by requiring the production to fire over more cycles to activate its target to a given level. Activation may also be conserved by deallocating some of the activation associated with previously activated elements, producing gradual forgetting.

Within this framework, the amount of demand that a task imposes on a resource pool can be defined as the number of units of activation required over a given time interval to perform the task. This quantity can be measured over the course of the model's performance of the task. Furthermore, the size of the supply of resources for a system is assumed to vary among normal individuals (due to practice or more physiological factors) and to be a source of individual differences in cognitive performance. Moreover, pathological neurological changes in an adult, such as those associated with strokes or dementia, may be viewed as reducing the resource supply (Miyake et al. 1994a, Haarmann et al. 1997).

Besides these assumptions about the resources, the theory also proposes a particular cognitive architecture that we will briefly describe here because it guides the interpretation of the research. $\uparrow$ The 3CAPS architecture is a hybrid system with both symbolic and connectionist features. The connectionist mechanisms have to do with the activation-based processing and storage that gives each function a graded quality and the potential for several processes to be executed in parallel. The symbolic mechanisms consist of the condition-action rules that express procedural knowledge and the production-system control regimen. If the activation of the elements in a condition side of a rule reaches a threshold, the production fires, incrementing the activation of the action elements. The processing is graded in the sense that production rules fire reiteratively, as long as their conditions are satisfied, usually until they attain their goal or some steady state. Thus, conditions and actions are linked by

$\dagger$ The 3CAPS system can be used for modelling diverse cognitive activities, including normal and aphasic language comprehension (Just and Carpenter 1992, Goldman and Varma 1995, Haarmann et al. 1997), mental rotation (Just and Carpenter 1995), reasoning (Carpenter et al. 1990, Just et al. 1996a) and human-computer interaction (Byrne and Bovair 1997, Huguenard et al. 1997). 
productions that propagate activation to action elements. The production rules are like dynamically formed links between nodes in a connectionist network (Carpenter and Just 1999) and self-schedule themselves based on the emergence of their enabling conditions, without any central control mechanism (such as a central executive). Furthermore, the processing can be parallel, in that all satisfied productions can fire concurrently in a given cycle if resources permit it; the processing can also be serial, if the initiation of some production is dependent on the activation of a product of some preceding computation.

\subsection{The concept of capacity utilization}

This framework provides a concrete operationalization of capacity utilization, a concept that was originally proposed in economics and refers to the proportion of resources that a system uses in a given time interval. For example, if a manufacturing plant operates for $8 \mathrm{~h}$ on a given day but is capable of operating a maximum of $24 \mathrm{~h}$ per day, then the capacity utilization is $33 \%$ for that day. Analogously, if an individual needs 15 units of activation to perform a cognitive task and has a total of 60 units of activation available, the capacity utilization for the duration of that task is $25 \%$. If a more difficult version of the task requires 30 units of activation, then it would have a capacity utilization of $50 \%$. Or, if an individual had a total of only 30 units available rather than 60 , the capacity utilization for a 30 unit task would be $100 \%$. These examples illustrate how capacity utilization is conjointly determined both by the amount of resources required by the task (i.e. demand) and the amount of resources available in the system (i.e. supply). In this article, we demonstrate that the concept of capacity utilization can be related to physiologically-based measures of brain activity obtained during cognitive tasks, including neuroimaging, pupil dilation and ERPs.

One important reason to turn to physiological measures of brain activity is that capacity utilization does not map directly onto traditional behavioural measures, such as response time or errors. Consider the issue of capacity utilization in the domain of mental arithmetic, in which one might compare a relatively easy but time consuming task of mentally adding a long list of single digit numbers $(3+2+5+2+3+1+4+6+1+\ldots=$ ?) versus a more demanding task (e.g. $63 \times 28=$ ?). The durations of the two tasks could be equated by choosing an appropriate list length for the addition task. Nevertheless, the addition task is postulated to draw on fewer resources for simultaneous processing and storage than does the multiplication task. The capacity theory, thus, predicts that there should be less brain activity in relevant areas during the addition task, even if the total durations of the two tasks are made equal.

The construct of capacity utilization focuses on a dimension that has been difficult to measure and that is often neglected in cognitive theory in spite of its importance: a characterization of how hard a cognitive system has to work to perform a given task (Just et al. 1996c). It seems introspectively obvious that our minds work much harder at some times than at other times. This notion of the mind's 'working harder' is concretized in the model, which provides mechanisms for mapping from processing characteristics to capacity utilization (as well as to response times and error rates). The mechanisms in the model that modulate capacity utilization during processing ultimately provide a measure of the total activation consumed during processing, which can be compared to the neuroindices of capacity utilization. 
Similarly, the mechanisms in the model that modulate the processing time provide measures of the time course of processing as well as the total processing time, both of which can be compared to behavioural measures obtained from human subjects. The model's processing time (the number of cycles of condition evaluations and subsequent firings) is determined partly by the capacity-constrained processing, such that the number of cycles required to perform some information operation is determined by resource availability, as is the determination of whether two or more processes are executed in parallel versus serially. Of course, a major determinant of processing time in the model is the number of processing steps that must be executed in sequence, which would be determined in the example above largely by the number of single digits to be added together.

We can use the example of the arithmetic tasks above to illustrate the concept of capacity utilization in the context of a 3CAPS model of the two contrasting tasks. In the single-digit addition task, we will ignore the need for a general addition algorithm and describe how one might calculate sums less than 100. The heart of this simple model would be the execution of several productions that would attempt to either retrieve or calculate the new running sum. The firing of a production would propagate activation to the new running sum over several cycles, with the amount being propagated per cycle being proportional to a parameter that links the condition and action. A verbal description of the productions is as follows:

(a) If there is a newly encoded digit (X) and a single-digit running sum

$\rightarrow$ then retrieve the sum of $X$ and running sum

(b) If there is a newly encoded digit (X) and a multi-digit running sum

$\rightarrow$ retrieve the sum of $\mathrm{X}$ and ones digit of running sum and maintain carry

(c) If there is a carry and partially revised sum

$\rightarrow$ then add the carry to the running sum's tens digit.

The difficulty of a sum retrieval process is partly a function of the frequency with which one has retrieved these facts in the past (Siegler and Shipley 1995). Thus, the frequency of the fact would affect the parameter that dictates the activation level of a condition element, which in turn determines how much activation is propagated to the action elements, with more activation being propagated per cycle if it is a more frequent fact. The difficulty is also affected by whether there is a need to calculate the sum (productions (b) and (c)) rather than retrieve them. For our addition example $(3+2+5+2+3+1+4+6+1+\ldots=?)$, most adults would probably be able to retrieve successive running sums with relatively little calculation.

In a 3CAPS model of mental multiplication, of the type required for calculating the answers to problems like $63 \times 28=$ ?, the total demand for activation is on average higher than in the single-digit addition task. Several productions would express the multiplication algorithm. It would involve activating to threshold several successive products, maintaining numerous intermediate results (i.e. the products of $3 \times 8,3 \times 2,6 \times 8$ and $6 \times 2$ ), activating the intermediate and final sums, and keeping track of where one is in the process. Because products are less frequently retrieved than sums, the individual retrievals would take longer than in the simple addition problem. More strikingly, the necessity of maintaining the intermediate products would also consume some activation. Consequently, the amount of activation consumed in a given time interval during the processing of this type of problem would be high. Thus, even though the two tasks could be designed to take the same amount of 
time, the prediction is that the resource consumption in the multiplication task would be greater than in the sequential addition task.

The 3CAPS theory focuses on capacity utilization at a particular system level that aggregates over lower-levels processes that undoubtedly exist and may even set parameters on the efficiency of processes at the higher-level. For example, the activation to threshold of a particular set of representations likely entails the inhibition of competing representations, although that is not yet incorporated into some of the 3CAPS models or in this mapping. The specific goal is to use the 3CAPS architecture to focus on the dynamics of resource modulation during immediate thought.

The theory postulates separate pools of resources for language processing and for spatial processing, based on numerous psychometric studies of cognitive abilities among normal young adults that find a separation of a verbal and a spatial factor (Carroll 1993, Shah and Miyake 1996). Furthermore, recent neuroimaging and neuropsychological studies of pre-frontal cortex suggest that there may be a third resource pool associated with executive processes (Duncan et al. 1996, 2000). Although the functional organization among these systems is unknown, it appears that the executive system plays a role in coordinating among systems, such as between the language and spatial systems. It may also play a role in maintaining a response over a delay period. Finally, it may play a role when a task is highly demanding for an individual, even if the task is entirely linguistic or entirely spatial. We describe the nature of the executive system in more detail below.

We further assume that different tasks can draw on each of the resource pools to different degrees. For example, language comprehension may primarily be supported by the language pool, and visuospatial reasoning may draw primarily on the spatial pool (Just and Carpenter 1992, Shah and Miyake 1996). However, just as discourse comprehension sometimes involves the construction of a spatial mental model of the situation (Friedman and Miyake 2000) and visuospatial reasoning tasks may sometimes invoke verbal strategies (Reid and Nygren 1988), many cognitive tasks are likely to draw on multiple resource pools to a different extent. In a 3CAPS model, there can be three resource pools of activation corresponding to these three systems. In this article, we do not need to specify the mapping between cognitive systems and tasks more precisely because we restrict our analysis to tasks that vary quantitatively while involving qualitatively similar processes. Thus, our primary focus is on slightly more demanding versus less demanding versions of the same type of task in either the linguistic, spatial or problem solving domain.

\subsection{Mapping 3CAPS activity onto neural processes}

The cortical neural systems corresponding to language, spatial and executive systems consist of several anatomically distinct regions. For example, language is partially subserved by cortical regions in the frontal and in the temporal lobes, more actively so in the left hemisphere, but also in the right. Each region may be further decomposable, but treating the language comprehension system as a single entity is a useful level of aggregation for our analysis. Furthermore, we make no claims about further decomposing or exhaustively enumerating the components of the systems. The anatomical correspondences are intentionally stated here without further precision because the critical mapping does not require (although it might benefit from) increasing anatomical precision.

The neural resources are assumed to consist of several diverse components, such as neurotransmitters' functioning, the various metabolic systems supporting the 
neural system, and also the structural connectivity or integrity of the neural systems. This view of the constituency of neural resources draws from the concept of neuralsystems efficiency of the type described by Parks et al. (1989), according to which the efficiency of a neural system is affected by the brain's neurochemistry, its cortical structural integrity, the topographical distribution of neuronal networks and the strategy (the actual cognitive processes that are executed). Parks et al. supported the general construct of neural system efficiency by showing a correlation between resting-state Positron Emission Tomography (PET) measures of glucose metabolism in Alzheimer patients and behavioural measures of performance in neuropsychological tests. Specifically, greater impairment was correlated with lower resting-state metabolic rate. Our interpretation of this finding is similar to Parks et al.'s, although the terminology is slightly different. We interpret the resting state PET measure as an index of the size of the potential resource supply: the lower the potential supply, the greater the impairment in the neuropsychological tests.

The current article examines the relation between resource consumption at the neural level and at the cognitive level, as specified by the 3CAPS architecture, for three cognitive systems. The claim is that for a certain range of task demand, there is a positive relation between the capacity utilization in a given cognitive system and the amount of metabolic activity. Thus, the amount of observed brain activity within this range should be related to resource consumption (such as rate or amount) relative to resource supply. We will examine three measures of brain activity that may serve as indices of capacity utilization in the brain. One is functional brain imaging, which relates to the amount of neural activation in a particular brain area and may reflect capacity utilization of neural systems within that area. The second is pupillary dilation, which may reflect an overall aggregate of capacity utilization that seems not to be system specific. (Although the neural basis of the link between the pupillary response and cognitive function is not yet known, current brain imaging technology should make it possible to determine the relevant neural substrate). The third is the relative magnitude of some ERP components, which may reflect the relative degree of capacity utilization. All three measures provide information about capacity utilization within large neural systems in a way that can be related to capacity utilization at the cognitive level.

\section{The language system}

The language comprehension system processes and coordinates the lexical, syntactic, thematic and pragmatic relations among a sequence of words and phrases to recover the meaning of their configuration. These processes emerge from a complex interaction between large-scale interconnected networks of brain areas. The classic characterization, based on clinical studies of aphasic patients tracing back to the time of Broca and Wernicke, primarily associates language production with Broca's area and language comprehension with Wernicke's area. Recent neuroimaging studies of the processing of verbal stimuli provide additional anatomical precision concerning a likely major function associated with some other cortical areas: auditory encoding is primarily associated with Heschl's gyrus (Binder et al. 1994), visual word-form encoding is primarily associated with the middle occipital gyrus and left extrastriate area (Howard et al. 1992) and lexical access may be primarily associated with the left middle temporal gyrus (Stowe et al. 1994), although it may be quite widely distributed in the left perisylvian region (Ojemann 1991). 


\subsection{Functional imaging of language processing}

Measures of functional brain activity, such as glucose metabolism rate or oxygen concentration, may provide a quantitative index of the amount of capacity utilization in a system or sub-system under certain circumstances. Of course, different metabolic measures have different properties (for reviews, see Haxby et al. 1991, Metter and Hanson 1994, Sergent 1994). For example, Blood Oxygen Level Dependent (BOLD) contrast in functional Magnetic Resonance Imaging (fMRI) measures neural activity via the increased flow of blood to the site of the computation. Thus, fMRI has slightly different properties than PET, which measures variation in the metabolism of glucose. These different physiological processes affect the time course and range over which the measure relates to the underlying neural activities. However, within these bounds, these measures may be quantitative indices that can relate to capacity utilization in a functional model of processing.

\subsubsection{Resource demand: complex sentence processing: As sentence comprehension} becomes more complex because of syntactic and semantic factors, the 3CAPS simulation requires more activation resources to perform the computations and to maintain the activated representations of the component word meanings, propositions and relational structures. The increases in activation in the model due to task demand correlates with increases in the amount of fMRI-measured brain activation (Just et al. 1996c). The amount of activation in four cortical areas (Wernicke's, Broca's, and their right hemisphere homologues) was examined in 15 normal right-handed adults, as a function of the demand imposed by the comprehension of three sentence types that are superficially similar (each containing two clauses and the same number of content words) but that differ in structural complexity and, hence, in the demand they impose (see table 1). Whereas type 1 sentences contain active clauses that are simply conjoined, the more complex type 2 sentences contain a relative clause that interrupts a main clause, causing additional maintenance. Finally, in type 3 sentences the main clause is interrupted, and the first noun plays different roles in the two clauses (as the subject of the main clause and the object of the relative clause).$\dagger$

The results, presented in figure 1 , show that the processing of more complex sentences leads to an increase in the volume of neural tissue that is highly activated in all four areas, Wernicke's area, Broca's area, and their right hemisphere homologues. We use the term Wernicke's area for convenience in referring to the posterior superior and middle temporal gyrus, and Broca's area for the inferior frontal gyrus, acknowledging that there are no clear cytoarchitectonic boundaries differentiating

Table 1. Three types of stimulus sentences differing in structural complexity.

(1) Active conjoined

(2) Subject relative clause

(3) Object relative clause
The reporter attacked the senator and admitted the error.

The reporter that attacked the senator admitted the error. The reporter that the senator attacked admitted the error.

$\dagger$ Supporting this complexity analysis, several studies have demonstrated that the most complex type (type 3, object relatives) produced longer processing times (King and Just 1991) and higher error rates (Miyake et al. 1994b) than the less complex type (type 2, subject relatives). 


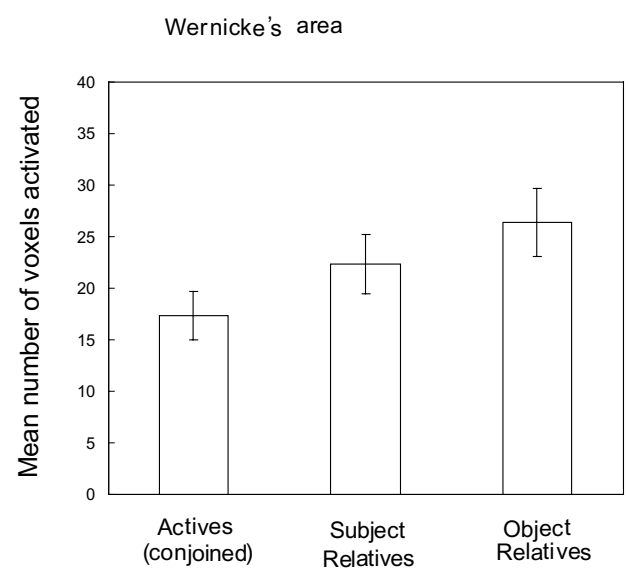

Right Homologue of Wernicke's area
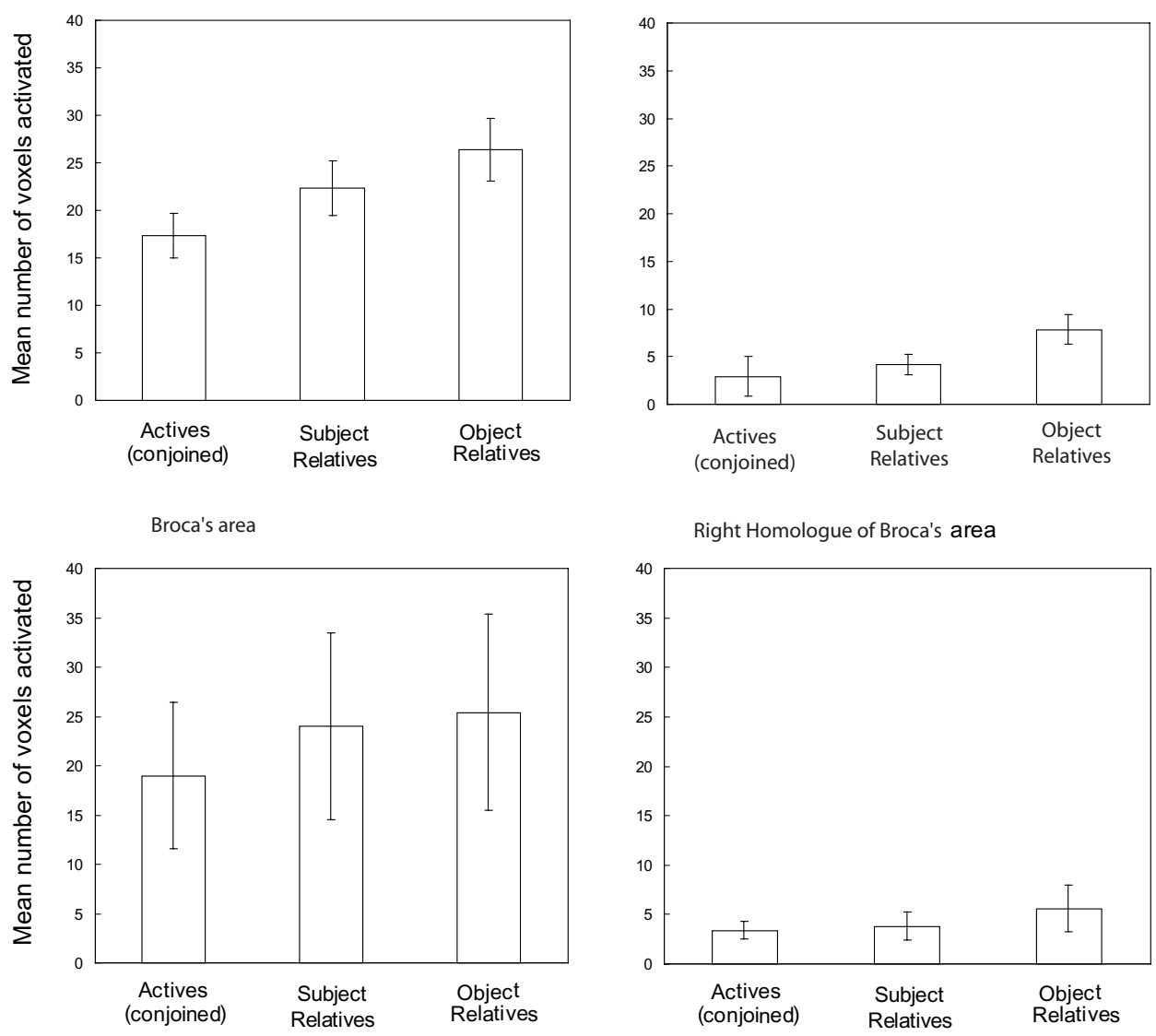

Figure 1. The top panels indicate the mean number of voxels activated per condition in the left (Wernicke's area) and right laterosuperior temporal cortex (and standard errors of the means over 15 participants). The bottom panels indicate the means in the left (Broca's area) and right inferior frontal cortex (and standard errors of the means over only five participants). From Just et al. (1996c: 115), copyright 1996 by the American Association for the Advancement of Science. Reprinted with permission.

these areas from those that surround them. Again, our emphasis is more on the functional relations than on localization, because the 'location' depends partly on the task demand. In this study, the demand is generated by computations beyond lexical processing (given that lexical content was equivalent across conditions), and such processes may include syntactic, thematic, referential processing, or some combination of them.

To illustrate the increasing level of activation within Wernicke's area, figure 2 shows a representative functional image from the most activated slice of one participant, superimposed on the structural brain image, showing in white the voxels that are significantly activated for each condition. This particular slice shows little of the activation in the right homologue or in Broca's area or its right-hemisphere homologue. Because the activation in Broca's area was difficult to evaluate precisely given the location of the axial scans, the activation was assessed in five of the 15 partici- 


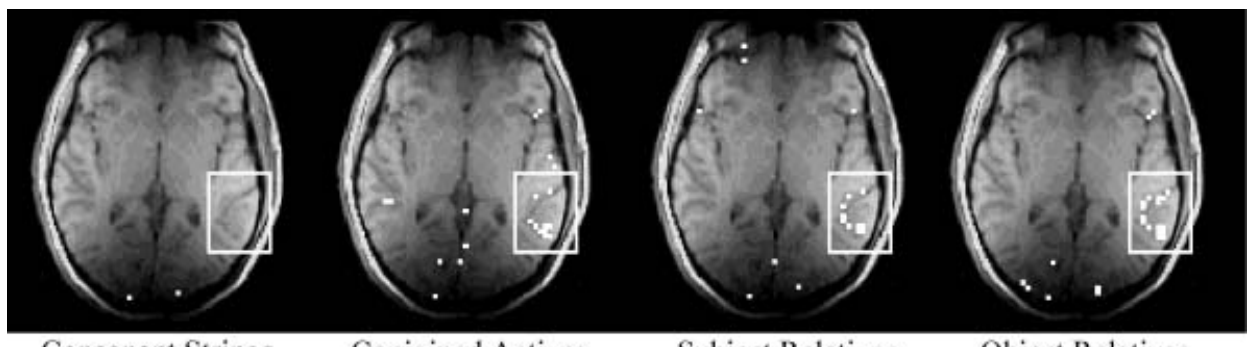

Consonant Strings

Conjoined Actives

Subject Relatives

Object Relatives

Figure 2. Thresholded fMRI brain activation images (superimposed on structural images) for one slice from one participant for each of four conditions compared to the rest condition. The voxels that are significantly activated in the left laterosuperior temporal cortex (Wernicke's area, indicated by the white rectangle) generally increase in number with sentence complexity (the left hemisphere is depicted on the right). From Just et al. (1996c: 115), copyright 1996 by the American Association for the Advancement of Science. Reprinted with permission.

pants (hence, the larger error bars) who were additionally scanned in an ancillary study using coronal slices and presenting comparable sentences. It has been suggested that this region might be the generator of the phonological code and/or it might be involved in syntactic processing, both of which may be required in comprehending these complex sentences. The modulated activation of the right homologue of Wernicke's area is consistent with the proposal that it, too, is part of the language network, in addition to being evoked in the processing of figurative (nonliteral) language (Bottini et al. 1994) and prosodic information (Tompkins and Mateer 1985). The modulated activation in four areas suggests that related computations may occur in a network of areas, including areas that are quite widely distributed. The important result for the current thesis is that the volume of fMRImeasured activation increases with the demand of the comprehension task in a number of cortical areas subserving language comprehension, consistent with the proposal that measures of the amount of brain activation reflect capacity utilization.

\subsubsection{Resource demand: brain activation in a language working memory task:} fMRI-measured activation is also related to the presence of another type of demand that is imposed in the context of the reading span task, which is intended to make simultaneous demands on processing and storage. The reading span task (Daneman and Carpenter 1980) requires individuals to comprehend a short sequence of sentences while maintaining an active representation of the sentencefinal words without covert rehearsal for recall. The fMRI study (Just et al. 1996b) compared activation in normal sentence reading to that in the span task, which requires additional maintenance. In a read-only condition, participants silently read and comprehended a short sequence of sentences. In a read-and-maintain condition, participants read and comprehended each sentence and, additionally, maintained the sentence-final words for later recall. The test assumes that the storage of the sentence-final words draws at least in part on the same resources as sentence processing. To ensure that the sentences were being comprehended and to decrease covert rehearsal, each sentence was verified as true or false, such as 'It is known that most people use their right hand to eat, write or drink'. As soon as the participant verified the sentence, the next sentence appeared for verification. 
The results from all four participants clearly supported the hypothesis that at least some of the same resources support sentence comprehension and storage of the final words. The read-only condition and the read-and-maintain condition both activated the same brain areas, namely Wernicke's and Broca's areas, the two major areas activated in the preceding fMRI comprehension study. In addition, the results supported the critical prediction that the amount of activation should be greater in one or more of these areas in the read-and-maintain condition than in the read-only condition because the demand on the common resource pool is greater when both comprehension and the ancillary maintenance are required. In all four participants, there was a reliably higher degree of activation in the read-and-maintain condition than in the read-only condition in Wernicke's area. Figure 3 shows that the number of reliably activated voxels in the most activated axial slice depicting Wernicke's area (boxed area) was greater in the read-and-maintain condition (the right hand column) for each of the four participants. Although the activation in Broca's area also increased somewhat, the increase was much smaller and less reliable than in Wernicke's area. Thus, the extra demand imposed by the reading span task results in more fMRI-measured activation in a brain area associated with sentence comprehension. This is consistent with the hypothesis that capacity utilization varies with task demand and that fMRI-measured activation can reflect capacity utilization.

The increase in activation in an area could be due to an increase in the number of activated neurons or increased activity in the same set of neurons. Both studies (Just et al. 1996b, c) provide evidence for both measures at this resolution. Moreover, there is no reason to think that the mechanisms are mutually exclusive. For example, it is known that intensity at the sensory level is coded by both an increase in the number of activated neurons as well as an increase in the firing rate of some neurons (Martin 1991). $\dagger$

3.1.3. Effects of resource supply: individual differences in language processing: The theory proposes that, in any given task, more skilled individuals should have lower capacity utilization and less brain activation than those with less skill, assuming that both groups can perform the task. The prediction stems from the assumption that a higher level of skill or ability effectively constitutes a larger resource supply. Supporting data come from a PET study of individual differences in a language-production task called verbal fluency, in which participants generate as many words as possible that begin with a given stimulus letter (Parks et al. 1988). The component processes in this task are not well specified, but they include generating candidate words, evaluating them, inhibiting the previously

$\uparrow$ As mentioned earlier, the theory's emphasis on activation should not be taken as a position on the role of inhibition, although it does raise the empirical question of how inhibition might be measured using either BOLD fMRI or PET metabolic indices of neuronal processing. Specifically, inhibition could be reflected as a decrease in blood flow, as Grasby et al. $(1993,1994)$ suggested when they observed systematic decreases in PET-measured blood flow in several areas in a digit span task. However, decreased blood flow could also reflect a redistribution of blood due to demand in other areas (Sergent 1994) or, in some cases, differences between experimental conditions that negatively covary with other processes. In any case, the theory's emphasis on activation reflects its lack of development with respect to inhibition. 


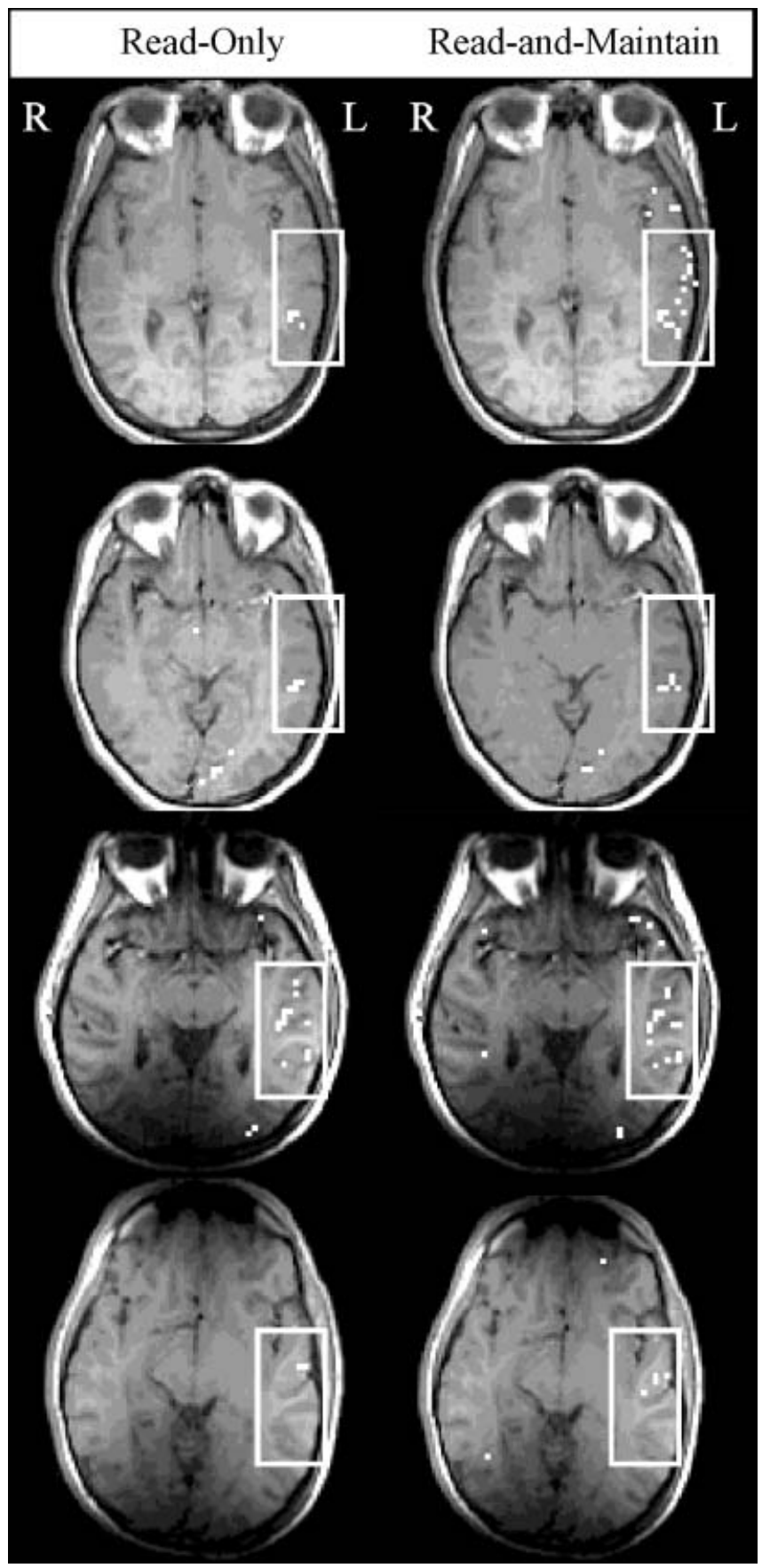

Figure 3. Thresholded fMRI brain activation images (superimposed on structural images) for one slice from each of four participants in the read-only condition (left-hand column) and in the read-and-maintain condition (right-hand column). The white rectangle highlights part of Wernicke's area, and the small white voxels are those that are significantly activated in that condition compared to the rest condition (the left hemisphere is depicted on the right). From Just et al. (1996b: 776), copyright 1996 by the American Psychological Association, Inc. Reprinted with permission. 
emitted items and searching for alternatives. These search and evaluation processes require resources for the component computations and storage and should be sensitive to capacity utilization. The central result of interest was that participants who were more proficient in the verbal fluency test (namely those who produced a greater number of correct words per unit time) also had lower levels of PET-measured brain activity (FDG uptake) in the relevant regions (bilateral temporal and frontal lobes). The capacity utilization proposal accounts for this result in terms of the resource supply that supports language processing. Participants with a larger resource pool consume a smaller proportion of that pool, resulting in less capacity utilization and, hence, less PET-measured neural activation.

\subsection{Pupil dilation during language processing}

Changes in the size of the pupil of the eye have been shown to reflect task demand during a variety of cognitive tasks (Kahneman 1973). The pupil starts to dilate gradually after the person encounters a significant cognitive demand, and this dilation peaks $\sim 1200 \mathrm{~ms}$ after the onset of the demand (Janisse 1977, Beatty 1982). The pupillary measure provides few clues about the locations of the underlying controlling neural systems. Instead, the measure may reflect a gross aggregate of local demand-supply relationships, whose aggregation mechanisms are not well understood. Also, pupillary measures may be less direct indicators of capacity utilization than functional brain activation measures, because the pupillary dilation during cognitive tasks has been hypothesized to be merely a correlate of resource demand; it may be a consequence of the cortical modulation of the reticular formation, which may also modulate some peripheral systems, including the pupillary control system (Beatty 1982).

3.2.1. Resource demand: Pupillary responses have been shown to be a sensitive index of processing demands that arise during sentence comprehension, where the increased demand is operationalized by a more complex sentence structure. Several studies have found a larger increase in pupil size for processing negative than for affirmative sentences and larger for syntactically anomalous than for well-formed sentences (Beatty 1982, Schluroff 1982). Pupillary responses are also sensitive to more subtle processing demand, such as the processing of multiple possible interpretations triggered by a lexical or syntactic ambiguity. Specifically, pupil dilation increases more when participants are reading sentences containing a lexical ambiguity compared to unambiguous sentences (Ben-Nun 1986) and in response to sentences containing a syntactically ambiguous pre-positional phrase compared to unambiguous sentences (Schluroff et al. 1986). The greater demand of lexically or syntactically ambiguous structures is also apparent in the reading time and comprehension accuracy (MacDonald et al. 1992, Miyake et al. 1994b). This parallel between the pupillometric data and the reading time and accuracy data suggests that resolving a linguistic ambiguity is a resource-dependent process, and that the pupillary response is sensitive enough to reflect this extra demand.

Syntactic complexity imposes another type of processing demand that evokes a greater pupillary response. In one study, the pupil size increase was greater if participants listened to more linguistically complex sentences compared to less complex sentences to paraphrase or memorize the sentences (Stanners et al. 1972). In our own experiment, we measured changes in pupil size as participants read simpler versus more complex sentences (Just and Carpenter 1993). The complex sentence types, 
object-relative sentences (such as The senator that the reporter attacked admitted the error) took longer to process than their simpler counterparts (such as The senator that attacked the reporter admitted the error) and elicited a greater change in the pupil diameter $(0.249 \mathrm{~mm}$ vs $0.203 \mathrm{~mm})$. A contribution of the study was to show that the increase in pupillary dilation was related to the increased processing at the point in the sentence where the reader encountered the syntactic complexity. In this way, the dilation measure also converged with increased gaze durations that were evoked at the same point in the sentence (although with a much shorter latency). Figure 4 graphically illustrates the mean pupil size during the sampled interval. $\uparrow$ These curves show that the maximum pupil size was larger during the reading of the more demanding object-relative sentences. In sum, changes in pupil size are sensitive to local resource demand imposed by the sentence comprehension.

\subsection{ERP measures of task demand}

The temporal resolution of ERPs is at least an order of magnitude finer than the resolution provided by brain imaging or pupillary measures. Moreover, ERPs pre-

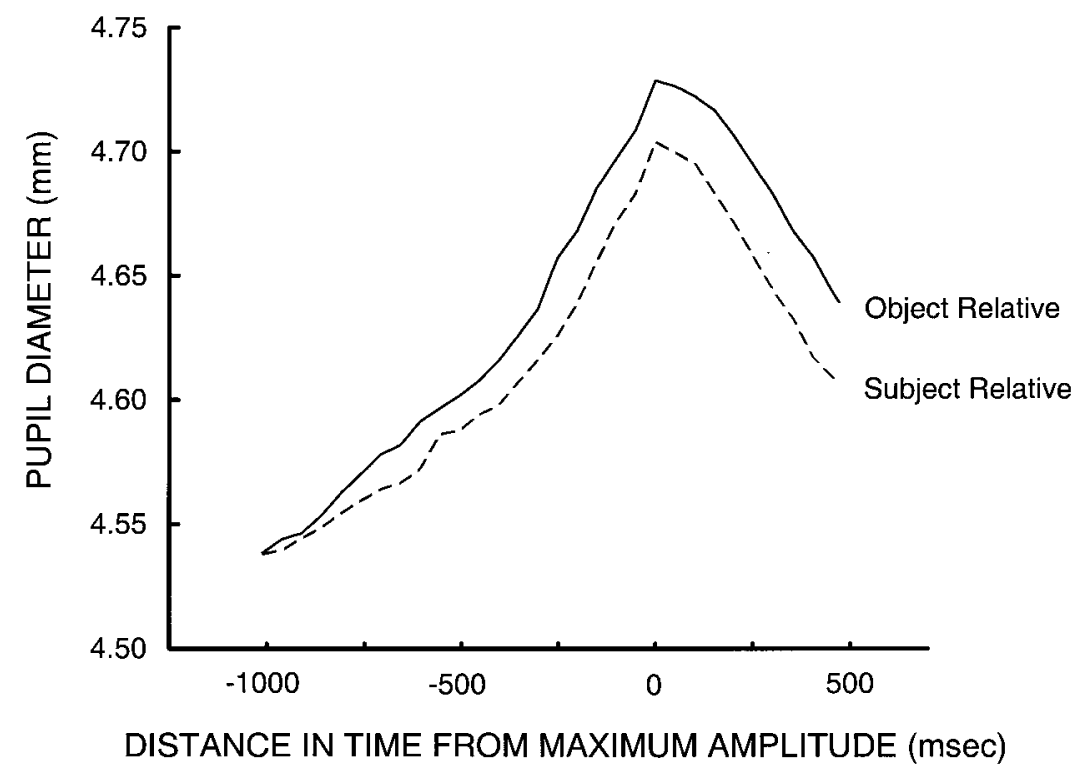

Figure 4. The pupil diameter for subject relative sentences and object relative sentences aligned according to the maximum amplitude. The curves indicate that the pupillometric dilation is greater during the comprehension of the more complex object relative sentences. From Just and Carpenter (1993: 320), copyright 1993 by the Canadian Psychological Association. Reprinted with permission.

$\dagger$ They were obtained by averaging over only a sub-set $(45 \%)$ of all the trials, excluding those trials $(19 \%)$ in which the maximum occurred within $113 \mathrm{~ms}$ (seven data samples) after the beginning of the interval and those in which $(36 \%)$ it occurred within $113 \mathrm{~ms}$ of the end of the interval. The averages were obtained by aligning the pupil size measurements at the point where the maximum occurred, and by including $1 \mathrm{~s}$ of data before the occurrence of the maximum and $550 \mathrm{~ms}$ after the maximum. An average waveform was computed for each participant in this way, and then an average of the subject averages was taken, and this grand average was smoothed, producing the curves in figure 4. 
sumably relate directly to neural electrical activity and, hence, they may provide an index of capacity utilization under some circumstances. However, there are other constraints that affect the measure's mapping to capacity utilization. One is that language processing may be somewhat altered by the typical experimental procedure of presenting the individual words of a sentence at a rate that is approximately twice as slow as normal reading (which is $\sim 250-300 \mathrm{~ms}$ per word). Moreover, an ERP waveform reflects a complex aggregate of both positive and negative electrical potentials generated by the cascaded underlying cortical processes whose spatial distributions are largely unknown. Consequently, the ERP studies of most interest in this article are those in which the experimental and control conditions involve qualitatively similar processes and which differ primarily quantitatively in the amount of processing or storage demand that the task imposes. This important qualification may permit us to spatially and temporally circumscribe the processes that are involved in a task without a more detailed model of the correspondence between specific ERP components and their functional correlates.

3.3.1. Resource demand: As was the case with some of the brain imaging and pupillometric studies, a number of ERP studies have compared the comprehension of relative-clause sentences that differ in complexity (Gunter et al. 1995, King and Kutas 1995, Mecklinger et al. 1995). For example, King and Kutas (1995) compared the ERPs elicited by reading object-relative and subject-relative sentences, such as The reporter who the senator harshly attacked admitted the error and The reporter who harshly attacked the senator admitted the error. The major differences in the ERPs were localized to the verbs, essentially the same sentence constituents that showed the major difference between the two sentence types. The ERPs at the verbs in the object-relative sentences showed more prolonged negativity than the ERPs at the verbs in the corresponding subject-relative sentences, particularly in the left anterior regions of the scalp. Moreover, better comprehenders showed less of the left anterior negativity associated with the relative clause than did poorer comprehenders, consistent with a difference in the neural effect of resource supply. $\dagger$

In examining the effect of processes that extend across multiple words, King and Kutas (1995) found a very slow positive drift over the frontal electrode scalp locations during the comprehension of the subject-relative sentences, but not objectrelative sentences. Moreover, the positivity was greater for good comprehenders than for poor comprehenders. According to Kutas and King (1996), this frontal positivity may be a cumulative index of the successful construction of a coherent sentence-level representation. The perspective provided by 3CAPS to such a result is that the construction and temporary maintenance of the sentence representation requires resources. While still largely conjectural, the consistent effects of task demand and individual differences indicates that ERPs can be useful in examining the capacity utilization associated with the comprehension of the sequence of words that constitute complex sentences.

$\uparrow$ The King and Kutas (1995) study separated good and poor comprehenders on the basis of their accuracy to the comprehension questions to the subject-relative and object-relative sentences used in the study. 
ERP data may illuminate the allocation of working memory resources within the language system to lower-level as compared to higher-level processes. Specifically, the ERP components that have been linked to changes in resource allocation (in the form of shifting attention) is the N1 and P2 complex. In general, N1 and sometimes P2 amplitudes are greater for attended stimuli than for unattended stimuli in both the auditory and visual modalities. It has been proposed that the N1 amplitude indexes the amount of resources devoted to processing an information channel that can be selected on the basis of spatial information. King and Kutas (1995) found substantial variations in the size of the N1-P2 complex that correlated with overall levels of comprehension, with poor comprehenders showing larger posterior N1-P2 amplitudes than good comprehenders in most cases. Poor comprehenders may have allocated more attention to visual processing or word encoding than did good comprehenders.

Other consistent findings concerning attentional allocation during language processing have been obtained in ERP studies that focused at the discourse level (Raney 1993, St George et al. 1994). For example, in St George et al.'s (1994) study, when participants read an ambiguous, vague passage either with or without a passage title that would clarify the meaning of the text, a larger P1-N1 component was elicited by the individual words when the title was present than when it was absent. This finding suggests that the presence of the title may have made high-level comprehension processes easier and, thereby, enabled the participants to allocate more resources to other aspects of the reading process, such as low-level word recognition and lexical access. In favour of this view, an index of high-level integration difficulty, namely the N400 component, was smaller when the title was present than when it was absent, showing the opposite pattern to the early P1-N1 component.

3.3.2. Resource supply: A recent ERP study also reports that the rapid, successful integration of semantic and syntactic information may be influenced, at least in part, by the availability of working memory resources as assessed by a correlate of the Daneman and Carpenter (1980) reading span task (Mecklinger et al. 1995). This study used young German college students as participants and compared the ERPs elicited during the reading of different right-branching relative-clause sentences containing either a semantically neutral verb or a verb that was semantically biased toward either an object-relative or subject-relative interpretation. The German sentence structures were such that the relative-clause verb always occurred after the two noun phrases that would serve as the agent and recipient of that action. The amplitude of the N400 observed on the neutral verb (in comparison to the semantically biased verb) was larger for high-span than low-span comprehenders, $\uparrow$ indicating that high-span readers anticipated the kinds of verbs that would likely follow these two noun phrases and, hence, had difficulty integrating the neutral verb with their running representation of the sentence. In other words, high-span readers may be able to take advantage of semantic constraints provided by the context to facilitate the analysis of syntactic information. This interpreta-

$\dagger$ The Mecklinger et al. (1995) study separated the participants primarily in terms of the speed with which they answered accompanying comprehension questions, but this measure was significantly correlated with working memory capacity, as measured by Daneman and Carpenter (1980) reading span test, $r=-0.51, p<0.05$. 
tion is consistent with the finding from reading-time studies that high-span readers are better than low-span readers at taking contextual information into account to guide their sentence interpretation (Just and Carpenter 1992).

In this section, we have reviewed how task demand and linguistic skill relate to three measures of brain activation. The claim is that aspects of these neural indices can be related to the resource consumption dimension in the 3CAPS architecture. In an earlier description of 3CAPS (Just and Carpenter 1992), we showed how increased complexity in sentence comprehension was associated with increased processing time, resulting in a processing time profile that corresponded closely to the reading time profiles obtained with college students. We now argue that capacity utilization is a related but separable dimension of the processing within the cognitive architecture. Moreover, it is this construal of capacity utilization that relates to neural indices of processing. When a higher proportion of the activation resources are needed for successful processing, there tends to be higher levels of activity in the associated large-scale cortical neural systems. A higher proportion of resources is needed when either the task is made quantitatively more demanding or if the individual is less skilled in that domain. In the next section, we will argue that these arguments hold not just for the language system but for the spatial processing system as well.

\section{The spatial processing system}

Spatial processing includes mental rotation, translation and other rigid and nonrigid spatial transformations that are commonly elicited in visual problem solving, such as psychometric tests of spatial ability. The theory proposes that the resources underlying visuospatial reasoning are somewhat separable from those supporting language processing in normal young adults, an assumption that is supported by numerous psychometric studies that yield domain-specific factors underlying verbal and spatial cognitive abilities for healthy young adults (Carroll 1993, Shah and Miyake 1996). We will discuss visually based tasks, although parts of the spatial system may also be activated through haptically encoded displays (Carpenter and Eisenberg 1978).

The brain organization for visuospatial processing includes several cortical areas, but we focus on two pathways that originate in the occipital area and have extensive connections in V2, V4 and V5, and then project forward. One pathway, the ventral stream, feeds into the inferior temporal lobe and is considered to be largely specialized for object recognition. Lesions in this area in monkeys and humans cause deficits in visual discrimination tasks, including face perception and visual object agnosia (Ungerleider and Haxby 1994). The second sub-system, the dorsal stream, connects to the parietal lobe and is involved in spatial analysis and processing (Ungerleider and Mishkin 1982). Lesions in this area are associated with visuospatial neglect, optic ataxia and difficulties in spatial learning, such as stylus maze learning (Ungerleider and Haxby 1994). Although there is a differentiation between the two pathways, they are extensively linked by known anatomical feedforward and feedback connections, suggesting that the two pathways might operate in conjunction with each other to accomplish many visual cognition functions. While we focus on these two networks, they do not exhaust the visuospatial system; for example, there are neuroanatomical connections between the visuospatial neural systems and the pre-frontal cortex. In delayed-match-to-sample tasks, activation occurs conjointly in the inferior parietal area and the pre-frontal area (in the region of the principal 
sulcus) of the monkey in tasks that involve maintaining spatial position (Friedman and Goldman-Rakic 1994). As in the case of the language system, the precise anatomical decomposition is not critical to our argument.

If neural activity reflects capacity utilization, then the performance of a more demanding visuospatial transformation task should entail more brain activity than a less demanding task. In addition, neural activation in this system should decrease with visuospatial skill. Within the visuospatial system, the normal individual differences in the functional resource supply can be assessed with psychometric spatial tests (Just and Carpenter 1985) and with a spatial span task that was constructed to be analogous to the reading-span task and that correlates highly with scores from spatial psychometric tests (Shah and Miyake 1996).

\subsection{Functional imaging of spatial processing}

4.1.1. Resource demand: An increase in the demand of certain types of visuospatial processes generally results in more brain activation in a network of areas, as assessed with various neuroimaging measures. A study that manipulated task demand in visuospatial processing was a mental rotation task in which some trial types required more rotation than others and which used magneto-encephalography (MEG) to measure the resulting modulation in brain activity (Michel et al. 1994). Here, demand is measured either in terms of the size of the rotation angle through which a representation must be mentally rotated or in terms of the processing of a computational model of the task performance (Just and Carpenter 1985). Although, strictly speaking, MEG is not a brain imaging technique in the same sense as PET or fMRI, this study reported two interesting results relevant to the 3CAPS theory. First, the duration of the MEG-measured cortical activity (suppression of activity in the $\alpha$-frequency range, namely $8-12 \mathrm{~Hz}$ ) was monotonically related to the size of the rotation angle. Thus, the more demanding the trial type was, the longer the MEG-measured activity persisted. Although this result converges with behavioural reaction-time measures (as well as the ERP data we will discuss later), it does not directly reflect on capacity utilization. The second interesting result was that the number of activated areas increased when the rotation angle was very large. Although most of the task-relevant brain activity was in the right hemisphere, additional areas (in the left hemisphere) became activated only when the rotation angle was very large. It is possible that when the spatialprocessing demand is very high, additional brain areas are brought into play, analogously to the result in the language comprehension study (Just et al. 1996c), showing increasing involvement in the non-dominant hemisphere as task difficulty increased. The MEG measurements, thus, indicate that both the duration and the location of cortical activity are sensitive to the task demand.

More recently, Carpenter et al. (1999) used fMRI to show that the activation volume in the parietal lobule increases with the size of the rotation angle in a Shepard-Metzler rotation task. Earlier neuroimaging studies had shown that activation occurs in the parietal region during mental rotation (Cohen et al. 1996, Alivisatos and Petrides 1997), but had not investigated how the cognitive workload affects the amount of activation. The resource-demand perspective of 3CAPS predicts that there should be an increase in fMRI-measured activation in the parietal regions as a function of the amount of mental rotation. The experimental conditions involved a graded manipulation of the angular disparity between the two ShepardMetzler figures, either $0^{\circ}, 40^{\circ}, 80^{\circ}$ or $120^{\circ}$, along with control (grid) condition in 
which subjects looked back and forth between corresponding cells of two grids. As predicted, there was a monotonic increase in the amount of parietal activation as a function of the increase in angular disparity. As the left-hand panel of figure 5 indicates, the number of significantly activated voxels (number of volume elements or voxels activated above a fixed threshold) in the parietal region, most of which were in and around the intraparietal sulcus, increased monotonically with angular disparity. According to the theoretical account, larger angular disparities require more resources for both computing more intermediate orientations and for maintaining representations of both stimuli being compared.

The results provide additional detail besides the monotonic relation between cognitive workload and brain activation. First, the amount of activation and effect of the rotation angle is similar in the left and right hemispheres, indicating the involvement of the parietal lobule of both hemispheres to similar degrees in this visuospatial task. The bilaterality is most asymmetric at $0^{\circ}$, where the right hemisphere is noticeably more responsive than the left, suggesting that bilaterality may increase with the task's demand, as it does in language processing. Secondly, the increased activation with rotation angle is manifested not only as an increase in the volume of activation (number of voxels activated), but also in their mean change in signal intensity (relative to a baseline fixation condition), as shown in the right-hand panel of figure 5. In summary, the cortical systems subserving spatial processing show increased activation with increased demand in mental rotation.

4.1.2. Resource supply: One way to systematically decrease the capacity utilization in a task is to provide practice that increases the participants' level of skill. If participants receive training or practice that improves their procedural knowledge,
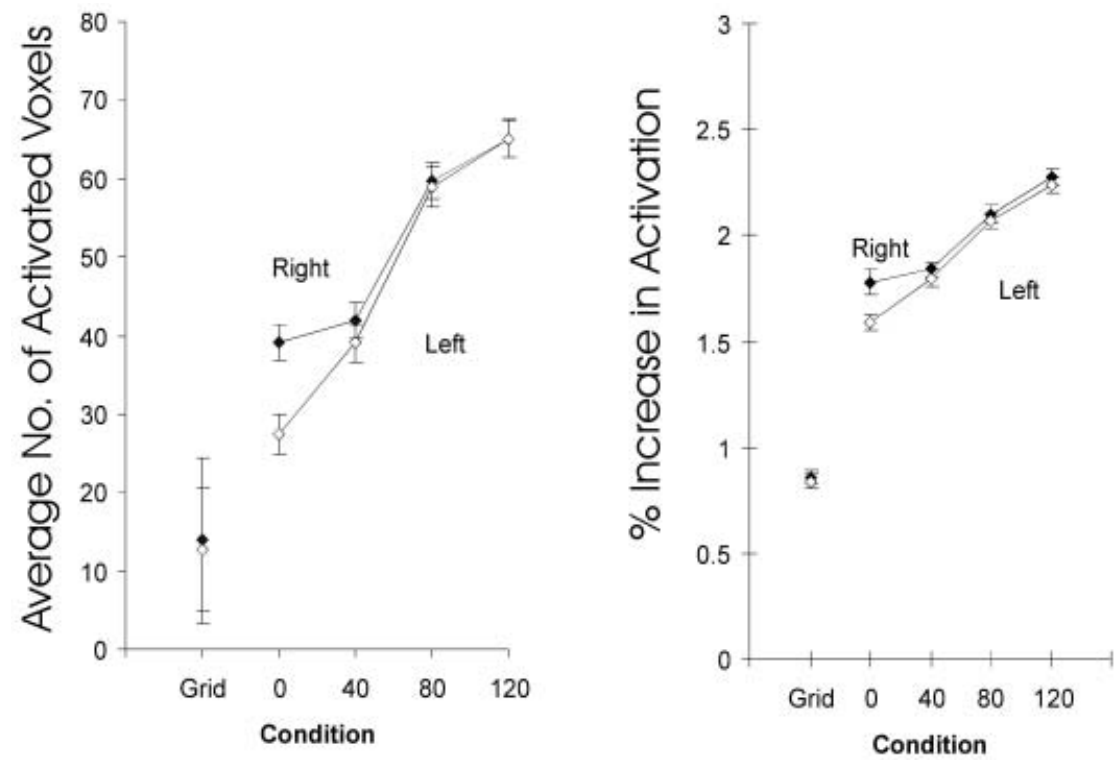

Figure 5. The average number of activated voxels for the left and right parietal regions during mental rotation increases as a function of the increase in angular disparity (left panel), as does the mean percentage of increase in MR signal intensity over the fixation condition (right panel). The results for the right hemisphere are shown by filled symbols and for the left by open symbols. 
they might be able to perform the task more efficiently, consuming a lesser amount of resources. Consistent with this prediction, a PET study (Haier et al. 1992) found that weeks of practice in the computer game Tetris not only led to improved performance in behavioural terms, but also reduced the amount of PET-measured activity (FDG-2-dyoxy-D-glucose labelled with F-18-uptake). It may be that there are new procedures acquired through practice that are less demanding of resources and, hence, entail a lower level of capacity utilization.

The facilitating effect of resource supply in visuospatial processing is consistent with a photon emission tomography (SPECT) study of individual differences in spatial ability (Charlot et al. 1992). Eleven participants were classified as high in spatial ability and 11 as low, as a function of having scored in the top third or bottom third of two widely-used psychometric tests of spatial ability. The experimental task required participants to visualize overflight between landmarks on a prememorized map. As the resource perspective predicts, the low ability group showed a substantial, reliable increase in SPECT-measured whole-cortex cerebral blood flow in the spatial imagery task (compared to a baseline), whereas the high ability group showed a much smaller (one third the size) and unreliable increase. (Examination of activity in specific cortical regions indicated that the task activation in the high ability group was more differentiated than in the low ability group). Thus, two groups that might be thought to differ in the supply of resources produced appropriately different whole cortex blood flow during the experimental task.

\subsection{Pupil dilation during spatial processing}

Changes in pupil size can also provide a useful index of capacity demand during spatial thinking tasks, such as mental rotation. In our own study (Just and Carpenter 1995), participants who were psychometrically classified as higher or lower in spatial skill judged whether a pair of irregular two-dimensional hexagons depicted the same figure (except for some disparity in orientation) or mirror-image figures, while their pupil sizes were being monitored. The two hexagons in a pair were presented at different orientations. A 3CAPS computational model of the process showed that, as angular disparity increases, successful performance requires more computation and storage, resulting in an increase in capacity utilization with degree of angular disparity. Consistent with the model, the pupillary response on the 'Same' trials increased monotonically as angular disparity increased. Secondly, the pupil size changes for low spatial participants were significantly greater than for the high spatial participants, indicating that the capacity utilization for the same operation was greater for individuals with less ability. Assuming that high spatial participants have more resources for spatial processing than low spatial participants, the difference between the groups is consistent with the interpretation that the ratio of demand to supply of resources may have been greater for the low spatial participants. These data suggest that the pupillary response is an index of capacity utilization, rather than an index of the absolute processing/storage demand placed on the participant.

A dynamic system, such as the 3CAPS cognitive simulation of mental rotation, provides several alternative indices of resource consumption that might plausibly correspond to the aggregate demand that is assessed by pupillary dilation. The function that best fit the pupillometric data across rotation conditions was the one that measured the proportion of the total activation that was consumed by the processing function of the model. In the absence of a more precise model of how 
pupillary dilation relates to cognitive demand, it is probably not worthwhile to more precisely determine the corresponding 3CAPS parameters. Nevertheless, the correspondence between activation consumption in 3CAPS and the pupillometric data supports the general claim of correspondence.

\subsection{ERPs during spatial processing}

4.3.1. Resource demand: ERP data can also serve as a useful index of capacity utilization during spatial thinking tasks, specifically during mental rotation (Peronnet and Farah 1989, Wijers et al. 1989, Desrocher et al. 1995). In these studies, ERPs were recorded while the participants judged whether a stimulus letter was normal or mirror-imaged. All three rotation studies found that as the angular disparity increased, there was increasingly more negativity in the ERP waveforms in the latency range of $350-800 \mathrm{~ms}$. Figure 6 illustrates this angular disparity effect observed in the Peronnet and Farah (1989) study. The graph plots the difference in ERP amplitude between the $0^{\circ}$ disparity condition and each of the $45^{\circ}, 90^{\circ}$, $135^{\circ}$ and $180^{\circ}$ rotation conditions in the latency range of $400-800 \mathrm{~ms}$. As the figure indicates, the amount of negativity in the ERPs shows a monotonic increase with increasing angular disparity, particularly for the parietal and occipital leads. Peronnet and Farah (1989) also found some amplitude differences in other latency ranges (i.e. some positivity in the $800-1200 \mathrm{~ms}$ range and further negativity in the 1200-1700 ms range), but these differences did not show systematic effects of angular disparity.

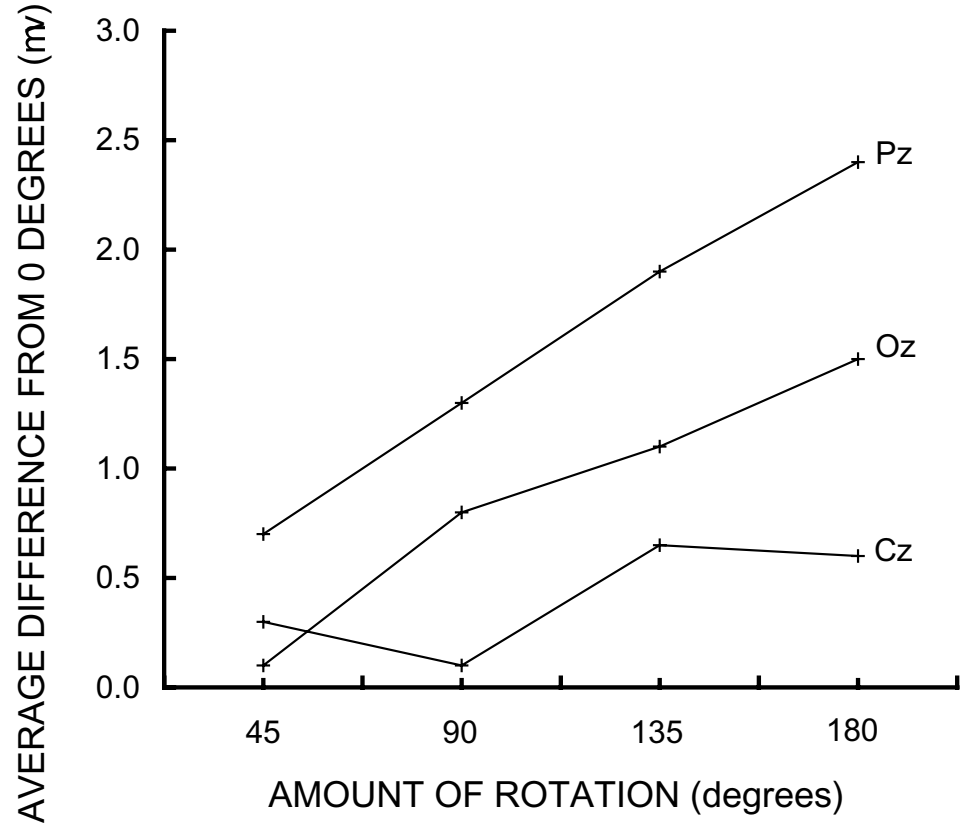

Figure 6. The increase in the waveform size in the range of 400-800 $\mathrm{ms}$ following stimulus presentation for the electrodes in the parietal $(\mathrm{Pz})$, occipital $(\mathrm{Oz})$ and central $(\mathrm{Cz})$ areas as a function of rotation condition compared to the $0^{\circ}$ condition. The measure is the area between the baseline condition waveform and the rotation condition waveforms. The monotonic increase is consistent with increasing effort as a function of angular rotation. From Peronnet and Farah (1989: 285), copyright 1989 by Academic Press. Reprinted with permission. 
Another ERP study used more complex stimuli to examine the patterns of electrophysiological activity during mental rotation (Rosler et al. 1995). In this study, participants were briefly ( $3 \mathrm{~s})$ shown a six-pointed star that consisted of 12 isosceles triangles, a few of which were filled. The star then disappeared, quickly followed by one of the three signal tones that indicated how much they should mentally rotate the star (either $0^{\circ}, 60^{\circ}$ or $120^{\circ}$ ). About $10 \mathrm{~s}$ after the onset of the tone signal, a probe star was presented, for which the participants were to decide whether the specified mental rotation was correctly performed or not. Despite the fact that this study examined a much larger temporal window (15s after the onset of the original star), the onset of the rotation process, signalled by one of the three tones, triggered pronounced negative potentials which extended over several seconds. The negative shift in slow waves was systematically related to the amount of mental rotation required, the more demanding rotation requirement inducing stronger negativity over the central and particularly the parietal leads. Such clear effects of angular disparity may again reflect the demand imposed on a network of cortical areas for visuospatial processing.

One interesting finding that emerges from these ERP studies of mental rotation concerns the sites of electrodes that demonstrate a clear, systematic effect of angular disparity. Central leads (Desrocher et al. 1995, Rosler et al. 1995) and occipital leads (Peronnet and Farah 1989) showed some systematic effects in a sub-set of the studies, but the effects were much more pronounced over the parietal leads in all four ERP studies discussed here. The distribution of the electrode sites showing rotation effects contrasts with ERP studies of language processing, which show that the frontal and central electrode sites show greater effects of syntactic complexity in language tasks like sentence comprehension (King and Kutas 1995). This difference in the distribution of electrodes that are sensitive to spatial and language tasks is consistent with the hypothesis that different neural circuits are recruited for spatial thinking and for language comprehension.

In sum, neuroimaging, pupillometric and ERP studies show increasing activation in the spatial system as a function of the task demand for mental rotation tasks, and they also provide evidence (although less extensive) that activation decreases with psychometrically-assessed spatial skill. These initial results are consistent with the proposed mapping of 3CAPS onto the visuospatial system. More generally, the convergence of the data in the visuospatial system suggest that a common set of principles may underlie the functioning of cognitive systems as disparate as the one that handles language comprehension and the one that handles visuospatial imagery. Furthermore, the results suggest that the concept of capacity utilization can be fruitfully mapped onto a diverse set of neurophysiological results.

\section{The executive system}

'Executive processes' refer to a constellation of meta-processes that enable relatively complex problem solving. They are executive processes in that they provide structure and organization to first order cognitive processes by generating and maintaining task goals and sub-goals (Carpenter et al. 1990), maintaining sequence and order information (Petrides 1995), inhibiting dominant but inappropriate responses (Roberts et al. 1994), helping to maintain information over time (Fuster 1997), switching back and forth between multiple tasks (Rogers and Monsell 1995) and integrating and coordinating information from multiple modalities (Emerson et al. 1999). Executive processes may enable the performance of complex tasks such as 
non-routine decision making, judgement and planning (Mesulam 1990), as well as performance in apparently much simpler tasks (such as delayed-match-to-sample) that require overcoming the normal tendency to respond immediately. In this section, we primarily focus on the executive system's role in the coordination of dual task performance and the planning and goal tracking in problem solving.

The same general principles proposed for the language and visuospatial processing systems may also apply to the executive system. Specifically, the amount of activation in the pre-frontal cortex should vary with the demand on executive processes and the supply of resources that an individual has available relative to the demand. At the behavioural level, the resources demanded for goal generation, maintenance and tracking has a large influence on the error rates for complex visual analogy problems (Carpenter et al. 1990). Also, consistent individual differences emerge as a factor in complex reasoning tasks, irrespective of the format or domain (visual, verbal, numerical) in which the problems are presented (Snow et al. 1984). The fact that some individual differences are consistent across formats or domains suggests the common variance may be a manifestation of a resource pool for executive processes, which works together with but is independent of the language and visuospatial systems.

The executive system has been identified primarily with the pre-frontal cortex for several reasons. First, the pre-frontal cortex has widespread connections with just about every type of paralimbic associative and cortical associative areas, including the language and visuospatial areas. By contrast, it does not receive direct sensory input the way the linguistic and spatial systems do, which is consistent with a more abstract role, such as coordinating and inhibiting systems that are functionally embedded within it (Mesulam 1990). Also, inferences about its overall contribution have been drawn from studies of neuropsychological patients who have lesions in the frontal lobe and show impairments in planning, abstraction and higher-level cognition (Shallice 1988). In addition to direct lesions of the pre-frontal area, the 'frontal syndrome' is often manifested by patients with multifocal white matter disease outside of the pre-frontal region (Mesulam 1990). This observation suggests that such dysfunctions can arise if there are sufficient impairments in the cognitive sub-systems that feed forward to and receive feedback from the pre-frontal system. Although the pre-frontal region itself can be further decomposed, neither the functional description nor the mapping to cortical circuitry are agreed upon, and this refinement is not crucial for the current theoretical point.

\subsection{Functional imaging of executive processing}

5.1.1. Resource demand: The theory predicts that the amount of task demand related to planning, sequencing and other executive processes should be associated with increased activation in the pre-frontal regions. In the realm of problem solving, it has been shown that the difficulty level of a Tower of London puzzle affects the amount of concomitant pre-frontal brain activation (Baker et al. 1996). The Tower of London puzzle requires participants to plan a sequence of several moves to transform a set of three objects (depicted as billiard balls) from one location to another with some constraints (e.g. no ball can be moved if there is a ball on top of it). The participants were able to plan easier problems (requiring two or three moves) in $\sim 5$ and $7 \mathrm{~s}$ on average, respectively, and harder problems (requiring either four or five moves) in 13 and $18 \mathrm{~s}$ on average, providing behavioural evidence of the relative difficulty of the various problem types. The main finding of 
interest for the capacity theory, however, concerns the comparison of brain activation in the easy and hard problems. The harder problems produced a significantly higher level of activation in the rostrolateral pre-frontal cortex, consistent with the hypothesis that the harder problems were more demanding of executive processes.

The amount of activation of the pre-frontal cortex in a short-term memory task showed sensitivity to increasing task demand (Braver et al. 1997). The Continuous Performance Task requires updating and maintaining sequence information as participants monitor for matches in a sequence of letters. As the targets occurred either 0,1 or 2 back, there was a monotonic increase in the amount of activation in the prefrontal cortex.

The involvement of the pre-frontal cortex in the coordination of dual-task performance was supported by the results of an fMRI study that contrasted dual task and single task performance. Even though the demand imposed by either one of two tasks alone (a semantic category judgement and a mental rotation task) was not sufficient to activate the dorsolateral pre-frontal cortex (DLPFC) (Brodmann's Areas 9 and 46), the demand imposed by having to perform the two tasks concurrently did (D'Esposito et al. 1995). More generally, the pre-frontal system may be evoked when the demands of a task are high and, hence, requires some strategic coordination of processing and storage even within a single sub-system. $\dagger$

Numerous studies have found activation in the pre-frontal region associated with short-term information maintenance. However, what is important for the current theoretical claim is that the activation showed sensitivity to the amount of demand (at least in the few studies that have parametrically varied the demand related to information maintenance, updating and goal generation). In addition to task demand, the other part of the capacity utilization equation has to do with resource supply, as reflected in cognitive skill. The evidence is beginning to accrue that cognitive skill is negatively related to activation in these types of tasks.

\subsubsection{Resource supply: A few studies that have examined individual differences} in brain activity in reasoning tasks have found increased activation for lower scoring individuals. One of the first imaging studies of cognitive individual differences (Haier et al. 1988) used FDG-PET to measure the brain activity of eight participants varying in their score on the Raven Progressive Matrices Test, which is a non-verbal, complex visual analogy task. One of the main determinants that distinguishes college students who score high in this test from those who score lower is the ability to generate and keep track of more problem solving sub-goals (Carpenter et al. 1990), which might relate to the size of the resource supply for executive processing. In the Haier et al. (1988) study, people with higher scores on the Raven test had lower levels of brain activity (cerebral glucose metabolic rate). The negative correlation held for activation in multiple regions, and was largest in the left and right temporal lobes, with correlations ranging from -0.73 to -0.92 in six temporal regions (Haier 1993). Although the brain imaging data are limited,

$\uparrow$ Consistent data for this proposal comes from a PET study of the verb generation task in which the demand was decreased by allowing the participants to practice generating the same verb to a noun stimulus until the task essentially required retrieval. After practice, the activation in DLPFC decreased such that it was no longer significantly activated (Raichle et al. 1994). 
owing to the small number of participants $(n=8)$ and to the non-independent assessment of capacity, the study provides remarkably compatible findings that are intuitively plausible but not easily encompassed by conventional cognitive theories that lack a dimension of intensity.

In sum, a few studies relating individual differences in cognitive ability to those in brain activity have reported higher levels of brain activity among participants of lower skill. Such studies are still limited by the cost of measuring the brain activity of a sizable number of participants. They are also limited by the lack of independent measures of performance that indicate that the higher- and lower-skill groups used the same strategy, as well as by the typical limitation that the evidence based on individual differences is correlational in nature and, thus, open to alternative interpretations. The available evidence, however, is quite consistent with the predictions derived from the capacity theory.

\subsection{ERPs during executive processing}

The availability of cognitive resources for executive processing has been studied in the context of human-factors studies of how task demand and participant characteristics affect dual task performance. In the 3CAPS framework, cognitive workload refers to the capacity utilization within the involved systems, including their coordination. Parasuraman (1990) summarized a number of ERP studies of the effects of cognitive workload in multitask situations and proposed that some components of the ERPs, particularly the P300 waveform, may provide an index of the relative resource allocation to each of the dual tasks. For example, participants might be asked to count the number of visual flashes that occur during a given interval, while simultaneously monitoring a visual display for changes in the direction of motion of the display elements (Isreal et al. 1980). The P300 amplitude for the secondary flashcounting task decreases as resources are deallocated from the secondary task to the primary task when the primary task is made more difficult. At the same time, the greater the amount of resources allocated to the primary task, the larger the amplitude of the P300 in response to a primary task event. The allocation of resources between dual tasks can also be manipulated through instructions and payoffs, and these manipulations also produce the expected effect on the amplitude of some component (not always the P300) of the ERP (Parasuraman 1985). Note that these results mean that the presence of a primary task can produce a decrease in the amplitude of the electrical response to the secondary task quite rapidly, within 300 ms of the stimulus. The P300 effect, thus, cannot easily be attributed to a deliberate (or controlled) strategic attempt to allocate resources after the stimulus has been presented.

Exactly how various ERP components correspond to the complex underlying cognitive processes is as yet not clear; nevertheless, these and earlier-mentioned studies suggest that there are systematic and interpretable effects of quantitative changes in task demand on ERP components. Perhaps one of the most interesting developments to emerge is the suggestion, both in these multitask situations and in the language comprehension domain (such as the studies of King and Kutas (1995)), that ERP measures provide indices of capacity utilization in the cascaded sub-processes that differ in their temporal profile. 


\section{General conclusions}

The interpretation of three disparate measures of neural activity (neuroimaging, pupillary dilation and ERPs) within a resource theory provides support for the main claim that there is a unifying construct, namely capacity utilization, that is an important metric of processing in tasks that stress immediate computation. The metric can be interpreted within a theory of the cognitive architecture, 3CAPS, that specifies both the types of representations and processes that are being computed and the nature of the resources that support those computations. The novel proposal is that these functional concepts of task demand and resource supply can be related to a variety of data on neural function in language comprehension, spatial processing and problem solving.

Before expanding on the implications of this approach, it is useful to reiterate some of its boundary conditions. One important boundary condition is that the tasks entailed some computation, such as language comprehension, visualization and problem solving. These immediate thought tasks constitute an operationalization of 'working memory', one which stresses the 'working' aspect of the construct (Just and Carpenter 1992, Miyake and Shah 1999). A second boundary condition is that the comparisons typically involve conditions that differ quantitatively in demand, while keeping the qualitative properties as similar as possible. A third boundary condition is that the task must be doable across the range of conditions with the same strategies by all individuals. If a task cannot be performed in one condition, or if it is performed by using different processes in different conditions, then the study no longer conforms to the assumption that the underlying processes differ quantitatively. However, if these boundary conditions are met, then some very interesting and novel claims can be made about the relation between the cognitive processes and the resulting neural indices.

One way of emphasizing the novelty of the current theoretical orientation, at least from the perspective of cognitive research, is by contrasting capacity utilization with temporal processing measures. Processing time measures certainly vie for the title of most common dependent measure in cognitive psychology, tracing back to at least the 1960s. The current theory proposes that processing time may be a useful index of demand, but that the relation is indirect because tasks that make little demand on resources per unit time can, nevertheless, take a long time. It is the claim of the current proposal that it is not time per se but capacity utilization that is more directly related to the neural activity. The emphasis on neural activity is not at all novel from the perspective of neuroscience, where researchers have long measured neural firing rates and hypothesized that they relate to behaviour. From this perspective, the novelty of the current proposal is the explicit link between a functional model of high-level cognition and the large-scale networks that perform the underlying cognitive computations.

The current analysis also supports the hypothesis that the supply of resources varies among normal individuals. In addition, pathological neurological changes (such as those associated with stroke and dementia, etc.) may also be construed as functionally reducing the resource supply, even though these neurological changes may correspond to a number of different neurobiological mechanisms. The changes in any of these mechanisms may reduce the efficiency of the neural circuitry that subserves some particular computation or computations. This theoretical view implies that it is possible to relate at least some aspects of the processing by neurological 
populations to the processing of unimpaired individuals (Miyake et al. 1994a, Haarmann et al. 1997).

It is difficult to compare the 3CAPS account of these data to that of some alternative cognitive architecture, simply because so few other architectures have explicitly addressed the quantitative aspects of brain activation. However, 3CAPS is a connectionist-type architecture, and it should be possible to generate isomorphic proposals using indices of demand in other connectionist architectures. The goal of the current article is not to argue that 3CAPS is the only answer; rather, the goal is to illustrate how this cognitive architecture provides a useful framework of at two levels of analysis for mapping between neural function and cognitive performance. A second point to raise is that 3CAPS is one member of an evolving family of architectures. Stimulated by the dynamics and quantitative properties of the fMRI data, we are currently developing a new generation of modelling environments that will address some of the multitude of issues raised by these data (for a preliminary report, see Just et al. 1999). These issues include the mechanisms underlying resource allocation, the detailed functional relations among cognitive sub-systems and their development. Thus, 3CAPS is only an approximation, but one that highlights the usefulness of considering an aggregate approach to cognitive sub-systems.

\subsection{Applications to human performance and ergonomics}

The theoretical approach provides not only scientific understanding, but also a set of analytical tools to measure and control cognitive workload. 3CAPS has previously proven itself useful in two human-computer interaction applications, using a purely behavioural approach, without regard to brain activity. One example is the work of Huguenard et al. (1997), which examined the use of a menu during a telephone-based interaction. Another example is the work of Byrne and Bovair (1997), who studied a frequent memory failure during the interaction with an ATM (automatic teller machine).

More recent work has used the successor theory to 3CAPS, namely 4CAPS (in which the 4th $\mathrm{C}$ stands for Cortical), to apply the approach to dual task performance (Just et al. 1999, 2001). The main purpose of the task was to examine brain activation during two high-level cognitive tasks that were intended to be representative of driving a car while using a cell phone. The actual tasks were mental rotation and verifying auditorily presented sentences as true or false. The study showed that the amount of brain activation associated with each of the two tasks was substantially lower (by 25-40\%) when each task was being performed concurrently with the other task, compared to when it was performed alone. To be sure, the behavioural performance also declined reliably, but not substantially, in the dual task situation. (It is important to note that when these two tasks were performed alone, they primarily activated different neural substrates, temporal and parietal areas, respectively. The non-overlap assures that any constraint on conjoint activation is not due just to competition for the same neural mechanisms.) Thus, there appear to be constraints on the conjoint functioning of multiple activated cortical areas. These system-wide resource constraints manifest themselves when subjects perform two high-level cognitive tasks concurrently. If there were no system-wide resource constraints, one would expect that the activation in a dual task would simply be the sum of the activations in each of the two single tasks, or even more than the sum (overadditive), because of additional overhead costs in performing both tasks simultaneously. However, the fMRI study clearly showed that the activation in the dual task was 
far less than the union of the two single tasks, indicating an overall resource constraint. The results are being replicated in which a computerized driving task is paired with auditory sentence verification, and the same results occur, increasing the likely generality of the findings to applied settings.

These results make it possible to calibrate levels of task demand in a dual task situation to the degree of interference mutually imposed by the two tasks. The system-wide constraints should apply when the brain activity in both tasks together approaches some upper limit. Using this perspective, it should be possible to determine at what level of traffic speed and density the language processing task affects driving-related brain activation. Similarly, it should be possible to determine what level of demand in the sentence processing task affects the brain activation associated with a given level of driving difficulty.

In summary, the current approach suggests that a fruitful level of analysis of workload is at the level of the cognitive system, which is orders of magnitude more molar than the neuronal level addressed by much of neuroscience. Even within cognitive systems, we have chosen a fairly high level, one that corresponds to the architectural and resource components needed to accomplish relatively complex cognitive activities, such as comprehending a sentence, rotating a visually presented display, or solving a complex analogy. From the perspective of cognitive neuroscience, this corresponds to very large-scale sets of interacting neural networks. These networks are apparently not localized in a small brain region, at least for most of the types of tasks described. This is not the only level at which mappings can be made between cognition and neural activity and, indeed, mappings have been proposed frequently at much finer levels and, sometimes, at more molar levels. However, the particular tasks examined have a time course and level of difficulty that is compatible with many cognitive paradigms and also with many neuroscience methodologies and, thus, may be particularly useful in the emerging field of neuroergonomics.

\section{Acknowledgements}

This research was supported in part by the Office of Naval Research Contract N00014-96-1-0322, and National Institute of Mental Health Research Scientist Awards MH00661 and MH00662. We would like to thank Erik Reichle and Vaibhav Didwadkar for their comments on the manuscript.

\section{References}

Alivisatos, B. and Petrides, M. 1997, Functional activation of the human brain during mental rotation, Neuropsychologia, 35, 111-118.

Baker, S. C., Rogers, R. D., Owen A. M., Frith, C. D., Dolan, R. J., Frackowiak, R. S. J. and Robbins, T. W. 1996, Neural systems engaged by planning: a PET study of the Tower of London task, Neuropsychologia, 34, 515-526.

BeAtTy, J. 1982, Task-evoked pupillary responses, processing load, and the structure of processing resources, Psychological Bulletin, 91, 276-292.

Ben-Nun, Y. 1986, The use of pupillometry in the study of on-line verbal processing: evidence for depths of processing, Brain and Language, 28, 1-11.

Binder, J. R., Rao, S. M., Hammeke, T. A., Yetkin, F. Z., Jesmanowicz, A., Bandettini, P. A., Wong, E. C., Estkowski, L. D., Goldstein, M. D., Haughton, V. M. and HYDE. J. S. 1994, Functional magnetic resonance imaging of human auditory cortex, Annals of Neurology, 35, 662-672.

Bottini, G., Corcoran, R., Sterzi, R., Paulesu, E., Schenone, P., Scapra, P., Frackowiak, R. S. J. and Frith, C. D. 1994, The role of the right hemisphere in 
the interpretation of figurative aspects of language: a positron emission tomography activation study, Brain, 117, 1241-1253.

Braver, T. S., Cohen, J. D., Nystrom, L. E., Jonides, J., Smith, E. E. and Noll, D. C., 1997, A parametric study of prefrontal cortex involvement in human working memory, NeuroImage, 5, 49-62.

Byrne, M. D. and Bovair, S. 1997, A working memory model of a common procedural error, Cognitive Science, 21, 31-61.

Carpenter, P. A. and Eisenberg, P. 1978, Mental rotation and the frame of reference in blind and sighted individuals, Perception and Psychophysics, 23, 117-124.

Carpenter, P. A. and Just, M. A. 1999, Computational modeling of high-level cognition, in R. J. Sternberg (ed.), The concept of cognition (Cambridge, MA: MIT Press), 245-293.

Carpenter, P. A., Just, M. A., Keller, T., Eddy, W. F. and Thulborn, K. R. 1999, Graded functional activation in the visuospatial system with the amount of task demand, Journal of Cognitive Neuroscience, 11, 9-24.

Carpenter, P. A., Just, M. A. and Shell, P. 1990, What one intelligence test measures: a theoretical account of the processing in the Raven Progressive Matrices Test, Psychological Review, 97, 404-431.

Carroll, J. B. 1993, Human cognitive abilities: A survey of factor-analysis studies (New York: Cambridge University Press).

Charlot, V., Tzourio, N., Zilbovicius, M., Mazoyer, B. and Denis, M. 1992, Different mental imagery abilities result in different regional cerebral blood flow activation patterns during cognitive tasks, Neuropsychologia, 30, 565-580.

Cohen, M. S., Kosslyn, S. M., Breiter, H. C., Digirolamo, G. J., Thompson, W. L., Anderson, A. K., Bookheimer, S. Y., Rosen, B. R. and Belliveau, J. W. 1996, Changes in cortical activity during mental rotation: A mapping study using functional MRI, Brain, 119, 89-100.

Daneman, M. and Carpenter, P. A. 1980, Individual differences in working memory and reading, Journal of Verbal Learning and Verbal Behavior, 19, 450-466.

D’Esposito, M., Detre, J. A., Alsop, D. C., Shin, R. K., Atlas, S. and Grossman, M. 1995, The neural basis of the central executive system of working memory, Nature, 378, 279-281.

Desrocher, M. E., Smith, M. L. and Taylor, M. J. 1995, Stimulus and sex differences in performance of mental rotation: evidence from event-related potentials, Brain and Cognition, 28, 14-38.

Duncan, J., Emslie, H., Williams, P., Johnson, R. and Freer, C. 1996, Intelligence and the frontal lobe: the organization of the goal-directed behavior, Cognitive Psychology, 30, 257-303.

Duncan, J., Seitz, R. J., Kolodny, J., Bor, D., Herzog, H., Ahmed, A., Newell, F. N. and Emslie, H. 2000, A neural basis for general intelligence, Nature, 289, 457-460.

Emerson, M. J., Miyake, A. and Rettinger, D. A. 1999, Individual differences in integrating and coordinating multiple sources of information, Journal of Experimental Psychology: Learning, Memory, and Cognition, 25, 1300-1321.

Friedman, H. R. and Goldman-Rakic, P. S. 1994, Coactivation of prefrontal cortex and inferior parietal cortex in working memory tasks revealed by 2DG functional mapping in the Rhesus monkey, Journal of Neuroscience, 14, 2775-2788.

Friedman, N. P. and Miyake, A. 2000, Differential roles for visuospatial and verbal working memory in situation model construction, Journal of Experimental Psychology: General, 129, 61-83.

Fuster, J. M. 1997, The prefrontal cortex: anatomy, physiology, and neuropsychology of the frontal lobe, $3^{\text {rd }}$ edn (Philadelphia: Lippincott-Raven).

Goldman, S. R. and VARMA, S. 1995, CAPing the construction-integration model of discourse comprehension, in C. A. Weaver, S. Mannes and C. R. Fletcher (eds), Discourse comprehension: Essays in honor of Walter Kintsch (Hillsdale, NJ: Erlbaum), 337-358.

Grasby, P. M., Frith, C. D., Friston, K. J., Bench, C., Frackowiak, R. S. J. and Dolan, R. J. 1993, Functional mapping of brain areas implicated in auditoryverbal memory function, Brain, 116, 1-20. 
Grasby, P. M., Frith, C. D., Friston, K. J., Simpson, J., Fletcher, P. C., Frackowiak, R. S. J. and Dolan, R. J. 1994, A graded task approach to the functional mapping of brain areas implicated in auditory-verbal memory, Brain, 117, 1271-1282.

Gunter, T. C., JaCkson, J. L. and Mulder, G. 1995, Language, memory, and aging: an electrophysiological exploration of the N400 during reading of memory-demanding sentences, Psychophysiology, 32, 215-229.

HaArmann, H., Just, M. A. and CARPenter, P. A. 1997, Aphasic sentence comprehension as a resource deficit: a computational approach, Brain and Language, 59, 76-120.

Haier, R. J. 1993, Cerebral glucose metabolism and intelligence, in P. A. Vernon (ed.), Biological approaches to the study of human intelligence (Norwood, NJ: Ablex), 317-332.

Haier, R. J., Siegel, B. V. Jr., Nuechterlein, K. H., Hazlett, E., Wu, J. C., Paek, J., Browning, H. L. and Buchsbaum, M. S. 1988, Cortical glucose metabolic rate correlates of abstract reasoning and attention studied with positron emission tomography, Intelligence, 12, 199-217.

Haier, R. J., Siegel, B., Tang, C., Abel, L. and Buchsbaum, M. S. 1992, Intelligence and changes in regional cerebral glucose metabolic rate following learning, Intelligence, 16, 415-426.

Haxby, J. V., Grady, C. L., Ungerleider, L. G. and Horwitz, B. 1991, Mapping the functional neuroanatomy of the intact human brain with brain work imaging, Neuropsychologia, 29, 539-555.

Howard, D., Patterson, K., Wise, R., Brown, W. D., Friston, K., Weiller, C. and FrackOWIAK, R. 1992, The cortical localization of the lexicons: positron emission tomography evidence, Brain, 115, 1769-1782.

Huguenard, B. R., Lerch, F. J., Junker, B. W., Patz, R. J. and Kass, R. E. 1997, Working memory failure in phone-based interaction, $A C M$ Transactions on Computer-Human Interaction, 4, 67-102.

Isreal, J. B., Wickens, C. D., Chesney, G. L. and Donchin, E. 1980, The event-related brain potential as an index of display-monitoring workload, Human Factors, 22, 211 224.

JANISSE, M. P. 1977, Pupillometry: The psychology of the pupillary response (Washington, DC: Hemisphere).

Just, M. A. and Carpenter, P. A. 1985, Cognitive coordinate systems: accounts of mental rotation and individual differences in spatial ability, Psychological Review, 92, 137-172.

Just, M. A. and Carpenter, P. A. 1992, A capacity theory of comprehension: individual differences in working memory, Psychological Review, 99, 122-149.

Just, M. A. and CARPEnTER, P. A. 1993, The intensity of thought: pupillometric indices of sentence processing, Canadian Journal of Experimental Psychology, 47, 310-339.

Just, M. A. and CARPenter, P. A. 1995, Spatial working memory: cognitive capacity in mental rotation, Unpublished Manuscript. Department of Psychology, Carnegie Mellon University, Pittsburgh, PA.

Just, M. A. and VARMA, S. 2002. A hybrid architecture for working memory: reply to MacDonald and Christiansen (2002), Psychological Review, 109, 55-65.

Just, M. A., Carpenter, P. A. and Hemphill, D. D. 1996a, Constraints on processing capacity: architectural or implementational?, in D. Steier and T. Mitchell (eds), Mind matters: A tribute to Allen Newell (Mahwah, NJ: Erlbaum), 141-178.

Just, M. A., Carpenter, P. A. and Keller, T. A. 1996b, The capacity theory of comprehension: new frontiers of evidence and arguments, Psychological Review, 103, 773-780.

Just, M. A., Carpenter, P. A., Keller, T. A., Eddy, W. F. and Thulborn, K. R. 1996c, Brain activation modulated by sentence comprehension, Science, 274, 114-116.

Just, M. A., Carpenter, P. A., Keller, T. A., Emery, L., Zajac, H. and Thulborn, K. R. 2001, Interdependence of non-overlapping cortical systems in dual cognitive tasks, NeuroImage, 14, 417-426.

Just, M. A., Carpenter, P. A. and Varma, S. 1999, Computational modeling of high-level cognition and brain function, Human Brain Mapping, 8, 128-136.

Kahneman, D. 1973, Attention and effort (Englewood Cliffs, NJ: Prentice Hall).

KING, J. and JUst, M. A. 1991, Individual differences in syntactic processing: the role of working memory, Journal of Memory and Language, 30, 580-602. 
KING, J. and KUTAS, M. 1995, Who did what and when? Using work- and clause-level ERPs to monitor working memory usage in reading, Journal of Cognitive Neuroscience, 7, 376-395.

Kutas, M. and KIng, J. 1996, The potentials for basic sentence processing: differentiating integrative processes, in T. Inui and J. L. McClelland (eds), Attention and performance $X$ VI: Information integration in perception and communication (Camgridge, MA: MIT Press), 501-546.

MacDonald, M. C., Just, M. and Carpenter, P. 1992, Working memory constraints on the processing of syntactic ambiguity, Cognitive Psychology, 24, 56-98.

Martin, J. H. 1991, Coding and processing of sensory information, in E. R. Kandel, J. H. Schwartz and T. M. Jessell (eds), Principles of neural science, $3^{\text {rd }}$ edn (New York: Elsevier), 329-340.

Mecklinger, A., Schriefers, H., Steinhauer, K. and Friederici, A. D. 1995, Processing relative clauses varying on syntactic and semantic dimensions: an analysis with eventrelated potentials, Memory and Cognition, 23, 477-494.

Mesulam, M.-M. 1990, Large-scale neurocognitive networks and distributed processing for attention, language and memory, Annals of Neurology, 28, 597-613.

Metter, E. J. and Hanson, W. R. 1994, Use of positron emission tomography to study aphasia, in A. Kertesz (ed.), Localization and neuroimaging in neurospychology (San Diego, CA: Academic Press), 123-149.

Michel, C. M., Kaufman, L. and Williamson, S. J. 1994, Duration of EEG and MEG a suppression increases with angle in a mental rotation task, Journal of Cognitive Neuroscience, 6, 139-150.

Miyake, A. and Shah, P. 1999, Models of working memory: Mechanisms of active maintenance and executive control (New York: Cambridge University Press).

Miyake, A., Carpenter, P. A. and Just, M. A. 1994a, A capacity approach to syntactic comprehension disorders: making normal adults perform like aphasic patients, Cognitive Neuropsychology, 11, 671-717.

Miyake, A., Just, M. A. and Carpenter, P. A. 1994b, Working memory constraints on the resolution of lexical ambiguity: maintaining multiple interpretations in neutral contexts, Journal of Memory and Language, 33, 175-202.

Navon, D. 1984, Resources - a theoretical soup stone?, Psychological Review, 91, 216-234.

NAvon, D. and Gopher, D. 1979, On the economy of the human processing system, Psychological Review, 86, 214-255.

Norman, D. A. and Bobrow, D. G. 1975, On data-limited and resource-limited processes, Cognitive Psychology, 7, 44-64.

OJemann, G. A. 1991, Cortical organization of language, Journal of Neuroscience, 11, 22812287.

Parasuraman, R. 1985, Event-related brain potentials and intermodal divided attention, Proceedings of the Human Factors Society, 29, 971-975.

PARASURAMAN, R. 1990, Event-related brain potentials and human factors research, in J. W. Rohrbaugh, R. Parasuraman and R. Johnson (eds), Event-related brain potentials: Basic issues and applications (New York, NY: Oxford University Press), 279-300.

Parks, R. W., Crockett, D. J., Tuokko, H., Beattie, B. L., Ashford, J. W., Coburn, K. L., Zec, R. F., Becker, R. E., McGeer, P. L. and McGeer, E. G. 1989, Neuropsychological 'systems efficiency' and positron emission tomography, Journal of Neuropsychiatry, 1, 269-282.

Parks, R. W., Lowenstein, D. A., Dodrill, K. L., Barker, W. W., Yoshit, F., Chang, J. Y., Emran, A., Apicella, A., Sheramata, W. A. and Duara, R. 1988, Cerebral metabolic effects of a verbal fluency test: a PET scan study, Journal of Clinical and Experimental Neuropsychology, 10, 565-575.

Peronnet, F. and Farah, M. J. 1989, Mental rotation: an event-related potential study with a validated mental rotation task, Brain and Cognition, 9, 279-288.

Petrides, M. 1995, Impairments on nonspatial self-ordered and externally ordered working memory tasks after lesion of the mid-dorsal part of the lateral frontal cortex in the monkey, Journal of Neuroscience, 15, 359-375. 
Raichle, M. E., Fiez, J. A., Videen, T. O., Macleod, A. K., Pardo, J. V., Fox, P. T. and Petersen, S. E. 1994, Practice-related changes in human brain functional anatomy during nonmotor learning, Cerebral Cortex, 4, 8-26.

RANEY, G. E. 1993, Monitoring changes in cognitive load during reading: an event-related brain potential and reaction time analysis, Journal of Experimental Psychology: Learning, Memory, and Cognition, 19, 51-69.

REID, G. B. and Nygren, T. E. 1988, The subjective workload assessment technique: A scaling procedure for measuring mental workload, in P. A. Hancock and N. Meshkati (eds), Human mental workload (Amsterdam: Elsevier), 185-218.

Roberts, R. J., Hager, L. D. and Heron, C. 1994, Prefrontal cognitive processes: working memory and inhibition in the antisaccade task, Journal of Experimental Psychology: General, 123, 374-393.

Rogers, R. D. and Monsell, S. 1995, Costs of a predictable switch between simple cognitive tasks, Journal of Experimental Psychology: General, 124, 207-231.

Rosler, F., Heil, M., Bajric, J., Pauls, A. C. and Henninghausen, E. 1995, Patterns of cerebral activation while mental images are rotated and changed in size, Psychophysiology, 32, 135-149.

SChluroff, M. 1982, Pupil responses to grammatical complexity of sentences, Brain and Language, 17, 133-145.

Schluroff, M., Zimmermann, T. E., Freeman, R. B., Hofmeister, K., Lorscheid, T. and Weber, A. 1986, Pupillary responses to syntactic ambiguity of sentences, Brain and Language, 27, 322-344.

Sergent, J. 1994, Brain imaging studies of cognitive functions, Trends in Neuroscience, 17, 221-227.

Shah, P. and Miyake, A. 1996, The separability of working memory resources for spatial thinking and language processing: an individual differences approach, Journal of Experimental Psychology: General, 125, 4-27.

Shallice, T. 1988, From neuropsychology to mental structure (New York: Cambridge University Press).

Siegler, R. S. and Shipley, C. 1995, Variation, selection, and cognitive change, in T. Simon and G. Halford (eds), Developing cognitive competence: New approaches to process modeling (Hillsdale, NJ:Erlbaum), 31-76.

Snow, R. E., Kyllonen, P. C. and Marshalek, B. 1984, The topography of ability and learning correlations, in R. J. Sternberg (ed.), Advances in the psychology of human intelligence, Vol. 2 (Hillsdale, NJ: Erlbaum), 47-103.

St. George, M., Mannes, S. and Hoffman, J. E. 1994, Global semantic expectancy and language comprehension, Journal of Cognitive Neuroscience, 6, 70-83.

Stanners, R. F., Headley, D. B. and Clark, W. R. 1972, The pupillary response to sentences: influences of listening set and deep structure, Journal of Verbal Learning and Verbal Behavior, 11, 257-263.

Stowe, L. A., Wijers, A. A., Willensen, A., Reuland, E., Paans, A. M. J. and VAALBURG, W. 1994, PET studies of language: an assessment of the reliability of the technique, Journal of Psycholinguistic Research, 23, 499-527.

Tompkins, C. A. and MAteER, C. A. 1985, Right hemisphere appreciation of prosodic and linguistic indications of implicit attitude, Brain and Language. 24, 185-203.

Ungerleider, L. G. and HaXby, J. V. 1994, 'What' and 'where' in the human brain, Current Opinion in Neurobiology, 4, 157-165.

Ungerleider, L. G. and Mishrin, M. 1982, Two cortical visual systems, in D. J. Ingle, M. A. Goodale and R. J. W. Mansfield (eds), Analysis of visual behavior (Cambridge, MA: MIT Press), 549-586.

Wickens, C. D. 1984, Processing resources in attention, in R. Parasuraman, and D. R. Davies (eds), Varieties of attention (New York: Academic Press), 63-102.

Wijers, A. A., Otten, L. J., Feenstra, S., Mulder, G. and Mulder, L. J. M. 1989, Brain potentials during selective attention, memory search, and mental rotation, Psychophysiology, 26, 452-467. 


\begin{abstract}
About the authors
Marcel Adam Just is DO Hebb Professor of Psychology at Carnegie Mellon University and a 15 year recipient of the NIMH Research Scientist Award. He has pioneered the use of new methods for tracing cognitive processes, including eye tracking, pupillometry and, more recently, neuroimaging and related detailed performance assessments using computational modelling. He is author of major theoretical and empirical publications on reasoning, cognitive modelling, language processing, visuospatial reasoning, individual differences and neuroimaging.
\end{abstract}

Patricia A. Carpenter is Lee and Marge Gregg Professor of Psychology at Carnegie Mellon University, recipient of NIMH Research Scientist Award and a member of numerous scientific, advisory and editorial panels. She is author of major theoretical publications on visuospatial reasoning, the nature and measurement of working memory, individual differences, language processing, eye fixations as a methodology to trace cognitive processes, neuropsychology, cognitive modelling and neuroimaging. She is a developer of measurement tools for assessing individual differences in working memory.

Akira Miyake is an Associate Professor of Psychology at the University of Colorado at Boulder. His research examines the nature and organization of working memory and executive functions and their roles in complex cognitive activities (such as language processing and learning, visuospatial thinking, problem solving and reasoning), using a variety of methodologies, including dual-task experiments, individual differences analyses and the testing of braindamaged patients. 